\title{
Changes In And Asymmetry of The Proteome In The Human Foetal Frontal Lobe During Early Development
}

\section{Shuwei Liu ( $\square$ liusw@sdu.edu.cn )}

School of Basic Medical Sciences, Cheeloo College of Medicine, Shandong University

\section{Xiaotian Zhao}

School of Basic Medical Sciences, Cheeloo College of Medicine, Shandong University

\section{Wenjia Liang}

School of Basic Medical Sciences, Cheeloo College of Medicine, Shandong University

\section{Wenjun Wang}

School of Basic Medical Sciences, Cheeloo College of Medicine, Shandong University

\section{Hailan Liu}

School of Basic Medical Sciences, Cheeloo College of Medicine, Shandong University

\section{Xiaolei Zhang}

Qilu Hospital of Shandong University, Cheeloo College of Medicine, Shandong University

Chengxin Liu

School of Basic Medical Sciences, Cheeloo College of Medicine, Shandong University

\section{Caiting Zhu}

School of Basic Medical Sciences, Cheeloo College of Medicine, Shandong University

\section{Baoxia Cui}

Qilu Hospital of Shandong University, Cheeloo College of Medicine, Shandong University

\section{Yuchun Tang}

School of Basic Medical Sciences, Cheeloo College of Medicine, Shandong University

\section{Article}

Keywords:

Posted Date: February 9th, 2022

DOl: https://doi.org/10.21203/rs.3.rs-1230789/v1

License: (c) (1) This work is licensed under a Creative Commons Attribution 4.0 International License.

Read Full License 
Version of Record: A version of this preprint was published at Communications Biology on September 29th, 2022. See the published version at https://doi.org/10.1038/s42003-022-04003-6. 
1 Changes in and asymmetry of the proteome in the human foetal frontal lobe

2 during early development

3

4

5 Author names

6

7

8 Affiliations

9

10

11 Corresponding author

12

13

14

15 
Hemispheric asymmetry is an important feature of the human brain that develops before birth. The inherent asymmetry of the brain is of great significance for cognition, language and other functions in humans. An understanding of normal brain and asymmetry development in the earliest period will further our understanding of how the different hemispheres prioritize specific functions. However, the core genetic mechanism is still unknown. Here, we analysed the developmental changes in and asymmetry of the proteome in the bilateral frontal lobes of three foetal specimens in the late first trimester of pregnancy. We found that during this period, the difference in expression between gestational weeks (GWs) increased, and the difference in asymmetric expression decreased. The patterns of protein expression changes in the bilateral frontal lobes were different. Our results show that brain asymmetry can be observed in the early stage of foetal development. Human brain asymmetry is the result of differential gene expression before birth in the early foetal stage and develops before functional asymmetry. Researchers can use these findings to further investigate the mechanisms of brain asymmetry. We propose that both sides of the brain should be analysed separately in future multiomics research and human brain mapping studies. 


\section{Introduction}

Asymmetry, a basic characteristic of the human brain and the brains of other vertebrates, was discovered in the mid-twentieth century ${ }^{1}$. At the population level, differences between the left and right hemispheres exist at almost all levels of the brain, including anatomical structure ${ }^{2}$, the connectivity of brain regions ${ }^{3}$, and function ${ }^{4}$. Brain asymmetry is critical for the maintenance of normal physiological functions in human, and changes in inherent brain asymmetry have been observed in ageing individuals ${ }^{5}$, in people with neurodegenerative diseases ${ }^{6}$ and in individuals with mental disorders ${ }^{7}$.

Brain structure asymmetry has been observed across the lifespan, including during early development before birth ${ }^{8,9}$. Foetal choroid plexus asymmetry can be observed in human foetuses from GWs 11 to $13^{10}$. It was also found that during this period, the movement of the right arm of the foetus is greater than that of the left arm ${ }^{11,12}$.

Because the prenatal brain is less affected by habits and environmental factors, the lateralization of brain structure and motor behaviour observed in the first three months of pregnancy is thought to be due to asymmetric genetic-developmental processes ${ }^{13}$. Genes that are differentially expressed between the left and right hemispheres have also been identified in previous studies. The developmental mechanism of brain lateralization from the middle stage of foetal development to adulthood has been explored. Recent genome-wide association studies (GWAS) have identified genetic loci that show strong associations with regional brain asymmetry ${ }^{14}$. Many genetic variants have been reported to be associated with adult human brain asymmetry at the 
genome level ${ }^{15-17}$. However, how brain asymmetry is established in the early stage of foetal development is not well understood.

Prenatal transcriptome data have revealed the widespread changes that occur during foetal development ${ }^{18,19}$, and a series of RNA sequencing studies have revealed the differential expression of genes between left and right hemisphere samples from embryos $^{20-24}$. However, when protein data was integrated with RNA-seq data, it was found that the difference in protein abundance between brain regions was generally higher than the difference in RNA levels ${ }^{25}$. Furthermore, a wide spectrum of genetic variants associated with diseases is often found at the proteome level. Differences in the expression of many proteins reflect the changes in cellular components and functions, and proteomic data provides direct information about the composition and functional states of proteins. However, given the limitation of study samples and technical limitations, these changes at the protein level remain unclear.

In addition to prenatal brain asymmetry, foetal brain development needs to be urgently studied since many psychiatric disorders in children and adults have been reported to originate before birth ${ }^{26,27}$. In recent years, researchers have extensively studied the development of the human cerebral cortex and the emergence of distinct neuronal lineages at the cellular level ${ }^{18,28}$. Despite this research, changes in the early foetal brain at the proteome level are not fully understood.

To explore the genetic-developmental mechanisms underlying typical brain asymmetry, we focused on the frontal lobes, which have been widely reported to exhibit structural asymmetry. We analysed asymmetrically expressed protein (AEP) 
groups and observed changes in an early stage of foetal development (GWs 9 to 13), a critical period for neuroblast proliferation and migration. Our study will help identify more sophisticated differences in the normal asymmetric development of the human brain.

\section{Proteins identified in the foetal frontal lobes at different $G W S$}

To investigate the protein compositions of the frontal lobe in early foetal development, we prepared tissue homogenates from the brains of human foetuses aborted at GWs 9, 11 and 13. The numbers of protein groups in the foetal frontal lobe included in the analyses after filtering were 3809 proteins in GW 9, 3905 proteins in GW 11 and 3661 proteins in GW 13. A total of 2645 protein groups were identified in all three GWs, as shown in the Venn diagram (Fig. 1a). Although more than 3000 proteins were detected in the bilateral frontal lobes at different GWs, a subset of protein groups (1793) was detected in all tissues (Fig. 1a). This core subset may be less affected by individual differences and may play a continuous role in human brain development. GO enrichment analysis revealed that the core proteins were enriched in the biological processes (FDR $<0.01$, Fisher exact test, Supplementary Fig. 1 and Supplementary Table 1) mRNA catabolic process, RNA catabolic process, RNA splicing and mRNA splicing, which occur widely throughout the development process.

To quantitatively compare the protein expression differences in tissues from different samples, we normalized the core protein data. We evaluated the effectiveness of various normalization methods (Supplementary Fig. 2) and chose CycLoess 
normalization ${ }^{29}$. Differentially expressed proteins (DEPs) between samples from different GWs were identified by limma with FDR control 0.05 using the normalized core protein subset. We identified 230 DEPs (out of 1793 proteins total (12.83\%); 114 upregulated and 116 downregulated) between GW 9 and GW 11 and DEPs 272 (15.17\%, 150 upregulated and 122 downregulated) between GW 11 and GW 13 (Fig. $1 \mathrm{~b}$ and Supplementary Table 2). With development, the number of DEPs increased, and the protein composition was more complicated, which is consistent with previous findings ${ }^{30,31}$. Among the DEPs, 69 core DEPs showed significant expression changes in two time periods (Fig. 1b). There was a significant protein-protein interaction (PPI enrichment $\mathrm{p}$ value: 0.0057 ) between these proteins (Fig. 1c and Supplementary Table 3). These core DEPs were significantly involved in mRNA binding (count $=8, \mathrm{FDR}=$ 0.016; purple), cell adhesion molecule binding (count $=11, \mathrm{FDR}=0.0081$; red) and RNA binding (count $=23, \mathrm{FDR}=2.88 \mathrm{e}-05$; green). It is worth noting that 8 proteins were enriched in the UniProt keyword mental retardation (count $=8, F D R=0.030$; yellow).

We identified multiple proteins that play a role in neuronal development and differentiation. The neuronal development factors that showed upregulated expression in GW 11 included neuronal cell adhesion molecule (NRCAM; $\log 2 \mathrm{FC}=1.09$, $\mathrm{FDR}=0.016)$, neuronal navigator $1(\mathrm{NAV} 1 ; \log 2 \mathrm{FC}=1.12, \mathrm{FDR}=0.012)$ and neurobeachin (NBEA; $\log 2 \mathrm{FC}=1.48, \mathrm{FDR}=0.0035)$. In addition, the expression of ten proteins associated with neurogenesis was significantly increased in GW 11 compared to GW 9, as identified by UniProt ID mapping (Supplementary Table 4). 
The most upregulated protein was cytoplasmic FMR1-interacting protein 1 (CYFIP1; $\log 2 \mathrm{FC}=2.34, \mathrm{FDR}=0.0016)$, which regulates cytoskeletal dynamics and protein translation $^{32}$, while the most downregulated protein was tubulin beta-2B chain (TUBB2B; $\log 2 \mathrm{FC}=-3.81, \mathrm{FDR}=0.00050$ ), which is a major component of microtubules ${ }^{33}$. In the comparison between the samples from GW 13 and the samples from GW 11, we identified 13 proteins associated with neurogenesis (Supplementary Table 5). The expression of NRCAM, a neuron development factor not associated with neurogenesis, increased $(\log 2 \mathrm{FC}=1.30, \mathrm{FDR}=0.0071)$, as in the comparison between samples from GW 9 and samples from GW 11. It is worth noting that the upregulated proteins neurofascin $(\mathrm{NFASC} ; \log 2 \mathrm{FC}=1.74, \mathrm{FDR}=0.0023)$ and contactin-1 $(\mathrm{CNTN} 1 ; \log 2 \mathrm{FC}=1.64, \mathrm{FDR}=0.0025)$ are related to axon growth. Moreover, the expression of Neurabin-2 (PPP1R9B; $\log 2 \mathrm{FC}=1.33, \mathrm{FDR}=0.0038$ ), which has been reported to be particularly highly expressed in dendritic spines ${ }^{34}$, increased with gestational age.

Through gene set enrichment analysis (GSEA) of the Gene Ontology gene sets, the biological processes that may be associated with the DEPs between different GWs were investigated. It was found that the expression of proteins associated with some key cellular processes related to cell adhesion, cellular component organization and macromolecule localization in the early stage of embryonic development, including cell-cell adhesion, organization of external encapsulating structure, and lipid localization, was downregulated from GW 9 to GW 11 (Fig. 2a; Supplementary Table 6). Interestingly, positive regulation of biological processes related to nervous system 
development, including cell projection organization, neuronal development, neurogenesis and neuronal differentiation processes, was evident at GW 13 compared to GW 11 (Fig. 2b and Supplementary Table 7). DEPs between GW 11 and GW 13 GW were more strongly related to neurodevelopment than those between GW 9 and GW 11.

We also explored protein expression differences between the GW 13 and GW 9 to assess more dramatic developmental changes with longer intervals. GSEA of the GO and KEGG data revealed that the top two enriched cellular components were neuronal projections $(\mathrm{NES}=0.42 ; \mathrm{FDR}=5.08 \mathrm{E}-07)$ and axons $(\mathrm{NES}=0.49 ; \mathrm{FDR}=1.25 \mathrm{E}-06)$ and that the top two enriched biological processes were neuronal development $(\mathrm{NES}=0.41 ; \mathrm{FDR}=6.65 \mathrm{E}-06)$ and nervous system processes $(\mathrm{NES}=0.55 ; \mathrm{FDR}=1.95 \mathrm{E}-$ 05) (Supplementary Table 8). This is consistent with the results of our analysis of developmental changes at two-week interval. DEPs between GW 9 and GW 13 were only significantly enriched in the DNA replication pathway (Fig. 2c), and KEGG pathway analysis showed that the DEPs identified at two-week intervals were not significantly enriched in any pathways.

To facilitate the probable assignment of cell types in the core protein dataset, three human foetal brain single-cell RNA-seq datasets were selected from the Molecular Signature Database (MSigDB). GSEA was performed to identify the overlapping DEPs between different ages in these cell type signature gene sets. In all three datasets, During development from GW 9 to GW 11, the DEPs were significantly enriched in brain endothelial cells (FDR $<0.001$ ), which were reported to 
appear beginning at GW $10^{35}$. The six most enriched cell types were nonneuronal cells (Fig. 2d), including pericytes and radial glia-like cells. Human microglial cell, human oligodendrocyte precursor cell (HOPC), human dopaminergic neuron (HDA) and human serotonergic neuron (HSERT), and human radial glia-like cell 2A (HRGL2A) signatures were significantly enriched in the GW 13 samples (Fig. 2d). OPCs are derived from neuroepithelial stem cells and can differentiate into oligodendrocytes $^{36}$, and endothelial cells likely extensively differentiate into OPCs at GW 11. This confirms our finding that protein expression differences at GWs 11-13 are more strongly associated with neural development. We hypothesized that these protein expression differences were due to the large number of mediolateral neuroblasts that differentiated into dopaminergic neurons. From GW 9 to GW 13, the most enriched cell type was microglia. The significant increase in the volume and complexity of the human cerebral cortex during this period is partly due to the exponential increase in the number of radial glia $^{37}$.

\section{AEPs in the foetal frontal lobes}

Proteome analysis of the frontal lobe at GW 9 yielded 2999 and 3524 protein groups that expressed in the frontal lobe. There were 2737 protein groups in the bilateral frontal lobes, and there were 3042 and 3089 protein groups the GW 11 samples, 2674 of which were shared, and 2744 and 3335 protein groups in the GW 13 samples, 2460 of which were shared (Fig. 3a). There were 3189 protein groups per frontal lobe on average. The complete results of peptide and protein identification for each GW are available in Supplementary Tables 18 (9 GW), 19 (11 GW) and 20 (13 GW). Six 
datasets from different GWs were subjected to principal component analysis (PCA) and Pearson correlation analysis to reduce the dimensionality of the array data and visualize the sample grouping. Samples from different GWs could be separated, indicating that the differences between hemispheres were more subtle than the differences between GWs (Fig. 3b).

We identified 81 protein groups ( 8 on the left side and 73 on the right side;

Supplementary Table 9, 10) expressed in only one side of the frontal lobe at three different GWs (Fig. 3c). The huntingtin (HTT) protein, which may play a role in microtubule-mediated transport or vesicle function, was among the proteins expressed only on the left side. Previous reports suggested that Huntington's disease affects the normal asymmetry of the human brain $^{38}$. By using UniProtKB keywords, we identified several consistent molecular functions associated with the protein groups expressed on only the left or right side (Fig. 3d and e), including hydrolase, activator, DNA binding and repressor. The proteins expressed only on the left side were not enriched in any molecular functions. The results revealed that proteins detected only in the right frontal lobe were also related to transferase, developmental protein, oxidoreductase, actin-binding, chaperone, calmodulin-binding, chromatin regulator, GTPase activation, translocase and isomerase (Fig. 3e). GO enrichment analysis showed that the proteins expressed on only the right side were enriched only in intracellular membrane-bound organelles $(\mathrm{FDR}=0.042$, count $=57$; Fig. $3 \mathrm{~g}$, red mark). This result indicated that the proteins that are expressed only on one side are mainly related to the process of foetal development. There was no significant 
interaction between the proteins expressed only in the left and right frontal lobes (Fig. $3 f$ and $g$ ), and the PPI enrichment $p$ values for the left and right frontal lobes were 0.535 and 0.396 , respectively. However, we believe that the interaction between these proteins has not been sufficiently studied.

AEPs between the left frontal lobe and right frontal lobe were considered significant when the fold change between the left frontal lobe and right frontal lobe was $>2$. Samples from the same GW were normalized using the label-free quantification were significantly highly expressed in the left frontal lobe, and 103 were significantly highly expressed in the right frontal lobe. At GW 11, 112 proteins were expressed on the left side and 40 being expressed on the right side (Supplementary Table 21-23). Interestingly, unlike the gradual increase in the number of DEPs during development, the number of AEPs between the left and right frontal lobes decreased with increasing gestational age (Fig. 4a). In previous studies, asymmetric expression of some genes was found to become less pronounced with increasing gestational age $^{22}$. We hypothesize that this result is consistent with previous reports of complex 231 changes in brain asymmetry in fetuses and infants ${ }^{39}$. We did not identify any proteins that showed a 2-fold change in expression at all three GWs, but 17 proteins were differentially expressed at two different GWs (Fig. 4b, c and Supplementary Table 11). These proteins included microtubule-associated 
proteins, i.e., tubulin beta-8 chain (TUBB8), tubulin alpha-1B chain (TUBA1B), microtubule-associated proteins 1A/1B light chain 3B (MAP1LC3B), and gammaaminobutyric acid receptor-associated protein (GABARAP). Recent studies have suggested that microtubule-related genes are related to brain structure asymmetry ${ }^{40}$. GWASs have revealed that TUBA1B is significantly associated with handedness and cortical surface area asymmetry ${ }^{41}$. Three accessory subunits of the mitochondrial membrane respiratory chain NADH dehydrogenase (Complex I), including NADH dehydrogenase [ubiquinone] 1 alpha subcomplex subunit 9 (NDUFA9), NADH dehydrogenase [ubiquinone] 1 alpha subcomplex subunit 10 (NDUFA10), and NADH dehydrogenase [ubiquinone] 1 alpha subcomplex assembly factor 2 (NDUFAF2), were also found to be associated with asymmetry. Previous studies have suggested that differential protein expression in the hippocampus occurs upon ageing and in Alzheimers disease ${ }^{42}$.

When a 1.5 -fold change was used as the screening criterion, 18 proteins, including NDUFA10, were found to be differentially expressed at all three GWs (Fig. 4d, e and Supplementary Table 12$)$. A total of $55.6 \%(10$ of $18, F D R=0.061)$ of the proteins were found to be associated with acetylation according to functional annotation (Supplementary Table 13). General transcription factor 3C polypeptide 1 (GTF3C1) was significantly overexpressed on the left side compared with the right side at three GWs, whereas annexin A1 (ANXA1), cellular retinoic acid-binding protein 1 
family 2, facilitated glucose transporter member 1 (SLC2A1) were underexpressed on

257

258 the left side.

GO enrichment analysis was performed to further elucidate the AEPs between the left and right frontal lobes at each GW. Fig. 5a-c show the top 10 significantly enriched GO terms in the three categories. AEPs at GW 9 were enriched in biological processes related to RNA splicing, mRNA splicing and growth factor (Fig. 5a and Supplementary Table 14). The upregulated proteins on the left side at GW 11 were associated with positive regulation of vascular permeability, and the downregulated proteins on the left side at this time point were associated with ATPase binding (Fig. 5b and Supplementary Table 15). We observed that the AEPs at GW 13 were mainly enriched in terms related to extracellular matrix, adhesion and binding and that the most significantly enriched GO terms were those enriched for proteins that were more highly expressed on the right side than on the left side (Fig. 5c and Supplementary Table 16).

To further analyse the candidate asymmetry-related genes, we aimed to assess the lateral expression profiles of protein groups in a more comprehensive manner. We performed GSEA of canonical pathway gene sets using the full ranked protein list to evaluate the biological role of the proteins that showed statistically significant differences in expression at each GW. The proteins that were highly expressed on the left side at GW 9 were associated with the metabolism of RNA (Fig. 5d). PRC2mediated methylation of histones and DNA, which is linked to epigenetic regulation, was the biological pathway for which the AEPs in right frontal lobe at GW 11 were 
enriched (Fig. 5e). GSEA strongly indicated that the NABA matrisome, which is assembled by genes encoding extracellular matrix (ECM) and extracellular matrixassociated proteins is the main canonical pathway affected by the proteins expressed in the right frontal lobe at GW 13 (Fig. 5f). During neural development, the ECM plays a key role in cell migration guidance, neural progenitor proliferation, neuronal morphology alterations, axonal projections and neural tissue morphogenesis ${ }^{43}$. GSEA of the ZHONG PFC gene set revealed significant enrichment of proliferating outer radial glia $(\mathrm{ORG})(\mathrm{FDR}=0.026)$ at $\mathrm{GW} 13($ Fig. $5 f)$ but no significant enrichment of any cell types at GW 9 or GW 11. The difference in cell types between samples from the two sides of the frontal lobe was not as significant as that between samples from different GWs.

\section{Asymmetry of protein changes in the bilateral frontal lobes during development}

To further understand the effect of development on frontal lobe asymmetry, we focused on the expression profiles of the 1793 core proteins, which were clustered into 16 profiles (Fig. 6a) using short time-series expression miner (STEM). We found that the expression of proteins in profile 15 in the frontal lobe was the same in both hemispheres (Fig. 6c and f). Notably, the expression of 50 proteins in profile 2 was found to be negatively correlated with age (Fig. 6b) in the left frontal lobe. Functional annotation clustering revealed that these proteins are predominantly involved in cellcell adherens junctions $(\mathrm{FDR}=0.045)$ and chaperone binding $(\mathrm{FDR}=0.00016$; Supplementary Table 17). The expression of proteins in profiles 12 and 13, which 
were upregulated to different extents, was significantly increased only on the right

301

302 side, and the expression of 52 protein clusters in profile 12 showed a significant linear correlation (Fig. 6d). The expression of 58 proteins in profile 13 appeared to increase faster with increasing gestational age (Fig. 6e). There may be subtle differences in the change patterns of protein expression in the bilateral frontal lobes during development. This finding confirms that there is a difference in the rate of development and maturity between the left and right brain ${ }^{21}$.

\section{Discussion}

In this study, we investigated the development of the human foetal frontal lobes from GW 9 to GW 13 and observed the difference in protein expression between the left and right frontal lobes. We found that during this period, the number of DEPs increased, while the number of AEPs decreased. Protein expression changes much faster during foetal development than during any other stage of life in humans $\mathrm{s}^{31,44}$. Transcriptome analysis has revealed found a sharp decrease in regional differences during late foetal development ${ }^{18}$. It has also been confirmed that the degree of asymmetry of brain network efficiency in young adults is significantly lower than that in adolescents ${ }^{45}$. The difference in protein expression between the left and right frontal lobes may be very difficult to detect in adults with normal physiological function $^{44}$. Our results show that the innate asymmetrical pattern of the brain changes and becomes less obvious with age.

It was found that multiple microtubule-associated proteins were among the core 
AEPs, which is consistent with the currently recognized phenomenon that the tubulin family controls organ asymmetry in many organisms by regulating cilia development ${ }^{46}$. In addition, we found that NADH dehydrogenase [ubiquinone] 1 alpha subcomplex subunits (NDUFA9, NDUFA10) and NADH dehydrogenase [ubiquinone] 1 alpha subcomplex assembly factor (NDUFAF2), which have not been reported to be related to asymmetric development, may be some of the key proteins in maintaining normal frontal lobe asymmetry in foetuses. Protein expression patterns in the frontal lobe differed between hemispheres with increasing gestational age, with the expression of a cluster of proteins showing a significant continuous downward trend on the left side but not on the right side.

The embryonic period ends at GW 8, when the basic structure of the brain and central nervous system has been established and the main sectors of the central and peripheral nervous systems have been identified. Neurons destined to form the neocortex, i.e., neuroblasts, are born beginning after the formation of the neural tube at GW 5, and the peak period of proliferation is from GW 6 to GW $18^{47,48}$. At GWs $12-20$, these neurons migrate along a scaffolding formed by glial cells ${ }^{49}$. The early changes in protein expression in the frontal lobe may be due to cell proliferation and differentiation. The foetal stage of human development, which is the key period for the development of the neocortex ${ }^{50}$, begins at GW 9, and brain development mainly involves the production, migration and differentiation of neurons. Our research results verify this conclusion. Compared with those between GW 9 and GW 11, the DEPs between GW 11 and GW 13 are more strongly associated with neurodevelopment. 
The proteins that showed downregulated expression between GW 9 and GW 11 were significantly enriched in cellular processes, while the proteins that showed upregulated expression from GW 11 to GW 13 were enriched in nervous system development, which represents the change from the embryonic to the foetal stage. Previous studies on the spatiotemporal distribution of cells in the human brain have shown that from early to middle foetal development, the number of progenitor cells decreases rapidly and the proportion and diversity of excitatory and inhibitory neurons increases; additionally, the number of astrocytes begins to increase in the middle stage of foetal development ${ }^{51}$. At the same stage, nonneuronal cells show few dynamic changes. Similar to this finding, in our study, the cell types in which DEPs were mainly enriched gradually switched from nonneuronal cells such as endothelial cells to mature neuronal cell types and glial cells from GW 9 to GW 13. Our findings provide a potential explanation for the dynamic changes in cell types in the human foetal frontal lobe, which helps us understand the cell types that change significantly during the early development stage.

However, we identified some proteins that showed significantly asymmetric expression in the foetal frontal lobes. Unlike the DEPs that were identified between different developmental time points, which were functionally similar, the AEPs identified between the left and right frontal lobes showed more subtle differences in both levels and associated biological processes. We also found no significant changes in the cell types that expressed the AEPs. From GW 9 to GW 13, the human brain is a smooth lissencephalic structure with no fissures except the cerebral longitudinal 
fissure, and at GW $14 \mathrm{GW}$, the Sylvian and cingulate fissures begin to appear ${ }^{52}$. The changes that occur during this short period, at which point brain morphology has been established but there is no evident cortical folding, are worthy of attention. However, few studies have focused on this period. The development of brain asymmetry is more refined and is likely not determined by one or a few regulatory pathways.

Furthermore, we believe that some proteins that are not significantly differentially expressed between the left and right brain may also play an indispensable role in the development of inherent asymmetry. We confirmed that the pattern of protein expression changes in the frontal lobe was different between the two hemispheres. Due to ethical limitations, it is difficult to completely preserve the morphology of the foetus during abortion. Samples that can be used for preliminary proteomics research are very precious. Due to this limitation of a small sample size, we excluded proteins that were not consistently detected for investigation of protein changes during development. We also chose best normalization method by comparing various standardized methods in an attempt to minimize the effect of individual differences on our results. Although imperfect, our study is the earliest age group to investigate the asymmetric protein expression in human brain tissue, which can provide some clues for future research.

As the brain is the most complex organ in the human body, the difference in protein expression between the left and right brain deserves further attention. More research on different brain regions, changes in protein expression at shorter intervals and single-cell proteomics will be important for elucidating the process of foetal brain 
388 development and the genetic mechanism of brain asymmetry. We propose that the two

389 sides of the brain should be analysed separately in future multiomics research and

390 human brain mapping studies. We will continue to pay attention to this issue in the 391 future.

392 


\section{References}

1. Güntürkün, O., Ströckens, F. \& Ocklenburg, S. Brain Lateralization: A Comparative Perspective. Physiol. Rev. 100, 1019-1063 (2020).

2. Kong, X. Z. et al. Mapping brain asymmetry in health and disease through theENIGMA consortium. Hum. Brain Mapp., (2020).

3. Shi, G. et al. The divided brain: Functional brain asymmetry underlying self-construal. Neuroimage. 240, 118382 (2021).

4. Goel, V. Asymmetrical involvement of frontal lobes in social reasoning. Brain. 127, 783-790 (2004).

5. Minkova, L. et al. Gray matter asymmetries in aging and neurodegeneration: A review and metaanalysis. Hum. Brain Mapp. 38, 5890-5904 (2017).

6. Wachinger, C., Nho, K., Saykin, A. J., Reuter, M. \& Rieckmann, A. A Longitudinal Imaging Genetics Study of Neuroanatomical Asymmetry in Alzheimer' s Disease. Biol. Psychiat. 84, 522530 (2018).

7. Kong, X. et al. Mapping Cortical and Subcortical Asymmetry in Obsessive-Compulsive Disorder: Findings From the ENIGMA Consortium. Biol. Psychiat. 87, 1022-1034 (2020).

8. Duboc, V., Dufourcq, P., Blader, P. \& Roussigné, M. Asymmetry of the Brain: Development and Implications. Annu. Rev. Genet. 49, 647-672 (2015).

9. Machado-Rivas, F. et al. Normal Growth, Sexual Dimorphism, and Lateral Asymmetries at Fetal Brain MRI. Radiology, 211222 (2021).

10. Abu-Rustum, R. S., Ziade, M. F. \& Abu-Rustum, S. E. Reference Values for the Right and Left Fetal Choroid Plexus at 11 to 13 Weeks. J. Ultras. Med. 32, 1623-1629 (2013).

11. McCartney, G. \& Hepper, P. Development of lateralized behaviour in the human fetus from 12 to 27 weeks' gestation. Dev. Med. Child Neurol. 41, 83-86 (1999).

12. Parma, V., Brasselet, R., Zoia, S., Bulgheroni, M. \& Castiello, U. The origin of human handedness and its role in pre-birth motor control. Sci. Rep.-UK. 7, (2017).

13. Francks, C. Exploring human brain lateralization with molecular genetics and genomics. Ann. $N y$. Acad. Sci. 1359, 1-13 (2015).

14. Sha, Z. et al. The genetic architecture of structural left - right asymmetry of the human brain. Nature Human Behaviour. 5, 1226-1239 (2021).

15. Carrion-Castillo, A. et al. Genetic effects on planum temporale asymmetry and their limited relevance to neurodevelopmental disorders, intelligence or educational attainment. Cortex. 124, 137-153 (2020).

16. Le Guen, Y. et al. Enhancer Locus in ch14q23.1 Modulates Brain Asymmetric Temporal Regions Involved in Language Processing. Cereb. Cortex. 30, 5322-5332 (2020).

17. Kong, X. et al. Large-Scale Phenomic and Genomic Analysis of Brain Asymmetrical Skew. Cereb. Cortex. 31, 4151-4168 (2021).

18. $\mathrm{Li}, \mathrm{M}$. et al. Integrative functional genomic analysis of human brain development and neuropsychiatric risks. Science. 362, (2018).

19. Lindsay, S. J. et al. HDBR Expression: A Unique Resource for Global and Individual Gene Expression Studies during Early Human Brain Development. Front. Neuroanat. 10, 86 (2016).

20. Ocklenburg, S. et al. Epigenetic regulation of lateralized fetal spinal gene expression underlies hemispheric asymmetries. ELife. 6, (2017).

21. de Kovel, C. G. F. et al. Left - Right Asymmetry of Maturation Rates in Human Embryonic Neural 
Development. Biol. Psychiat. 82, 204-212 (2017).

22. Sun, T. et al. Early Asymmetry of Gene Transcription in Embryonic Human Left and Right Cerebral Cortex. Science. 308, 1794-1798 (2005).

23. Miao, N. et al. Differential expression of microRNAs in the human fetal left and right cerebral cortex. Mol. Biol. Rep. 47, 6573-6586 (2020).

24. de Kovel, C. G. F., Lisgo, S. N., Fisher, S. E. \& Francks, C. Subtle left-right asymmetry of gene expression profiles in embryonic and foetal human brains. Sci. Rep.-UK. 8, (2018).

25. Carlyle, B. C. et al. A multiregional proteomic survey of the postnatal human brain. Nat. Neurosci. 20, 1787-1795 (2017).

26. Haniffa, M. et al. A roadmap for the Human Developmental Cell Atlas. Nature. 597, 196-205 (2021).

27. Barnat, M. et al. Huntington's disease alters human neurodevelopment. Science. 369, 787-793 (2020).

28. Ziffra, R. S. et al. Single-cell epigenomics reveals mechanisms of human cortical development. Nature. 598, 205-213 (2021).

29. Välikangas, T., Suomi, T. \& Elo, L. L. A systematic evaluation of normalization methods in quantitative label-free proteomics. Brief. Bioinform. 19, w95 (2018).

30. Kang, H. J. et al. Spatio-temporal transcriptome of the human brain. Nature. 478, 483-489 (2011).

31. Colantuoni, C. et al. Temporal dynamics and genetic control of transcription in the human prefrontal cortex. Nature. 478, 519-523 (2011).

32. Oguro-Ando, A. et al. Increased CYFIP1 dosage alters cellular and dendritic morphology and dysregulates mTOR. Mol. Psychiatr. 20, 1069-1078 (2015).

33. Guerrini, R. et al. Symmetric polymicrogyria and pachygyria associated with TUBB2B gene mutations. Eur. J. Hum. Genet. 20, 995-998 (2012).

34. Meng, X. et al. PPP1R9B (Neurabin 2): Involvement and dynamics in the NK immunological synapse. Eur. J. Immunol. 39, 552-560 (2009).

35. La Manno, G. et al. Molecular Diversity of Midbrain Development in Mouse, Human, and Stem Cells. Cell. 167, 566-580 (2016).

36. Spitzer, S. O. et al. Oligodendrocyte Progenitor Cells Become Regionally Diverse and Heterogeneous with Age. Neuron. 101, 459-471 (2019).

37. Huang, W. et al. Origins and Proliferative States of Human Oligodendrocyte Precursor Cells. Cell. 182, 594-608 (2020).

38. Mühlau, M. et al. Striatal gray matter loss in Huntington's disease is leftward biased. Movement Disord. 22, 1169-1173 (2007).

39. Liu, T. et al. Diffusion MRI of the infant brain reveals unique asymmetry patterns during the firsthalf-year of development. Neuroimage. 242, 118465 (2021).

40. Sha, Z. et al. The genetic architecture of structural left - right asymmetry of the human brain. Nature Human Behaviour. 5, 1226-1239 (2021).

41. Cuellar-Partida, G. et al. Genome-wide association study identifies 48 common genetic variants associated with handedness. Nature Human Behaviour. 5, 59-70 (2021).

42. Zhang, L. et al. Potential hippocampal genes and pathways involved in Alzheimer's disease: a bioinformatic analysis. Genet Mol Res. 14, 7218-7232 (2015).

43. Humphrey, J. D., Dufresne, E. R. \& Schwartz, M. A. Mechanotransduction and extracellular matrix homeostasis. Nat. Rev. Mol. Cell Bio. 15, 802-812 (2014).

44. Pletikos, M. et al. Temporal Specification and Bilaterality of Human Neocortical Topographic Gene 
Expression. Neuron. 81, 321-332 (2014).

45. Zhong, S., He, Y., Shu, H. \& Gong, G. Developmental Changes in Topological Asymmetry Between Hemispheric Brain White Matter Networks from Adolescence to Young Adulthood. Cereb. Cortex. 27, w109 (2017).

46. Inaki, M., Liu, J. \& Matsuno, K. Cell chirality: its origin and roles in left-right asymmetric development. Philos Trans R Soc Lond B Biol Sci. 371, (2016).

47. Spencer-Smith, M. \& Anderson, V. Healthy and abnormal development of the prefrontal cortex. Dev. Neurorehabil. 12, 279-297 (2010).

48. Chini, M. \& Hanganu-Opatz, I. L. Prefrontal Cortex Development in Health and Disease: Lessons from Rodents and Humans. Trends Neurosci. 44, 227-240 (2021).

49. Lenroot, R. K. \& Giedd, J. N. Brain development in children and adolescents: Insights from anatomical magnetic resonance imaging. Neuroscience \& Biobehavioral Reviews. 30, 718-729 (2006).

50. Stiles, J. \& Jernigan, T. L. The Basics of Brain Development. Neuropsychol. Rev. 20, 327-348 (2010).

51. Song, L. et al. STAB: a spatio-temporal cell atlas of the human brain. Nucleic Acids Res. 49, D1029D1037 (2021).

52. Zhan, J. et al. Spatial - temporal atlas of human fetal brain development during the early second trimester. Neuroimage. 82, 115-126 (2013). 


\section{Ethical approval and consent to participate}

Brain tissue was collected from foetuses discarded following induced pregnancy termination at Qilu Hospital, usually within 2 hours of the procedure. The medical staff involved in conducting the pregnancy termination procedures were not involved in this scientific study. The Ethics Committee of Shandong University School of Basic Medical Sciences approved the study, and all participants provided written informed consent. All clinical information was collected, and the donors had no psychiatric disorders or a family history of psychiatric disorders. Ultrasonography was used to confirm that the foetal samples did not exhibit intracranial pathology.

\section{Sample preparation, protein expression quantification and SDS-PAGE}

Discarded brain tissues of GW 9, 11 and 13 foetuses were collected. The whole brains were harvested, and the left and right cerebral hemispheres were separated. Each hemisphere was dissected immediately with a scalpel, and tissues from the front part of the frontal lobes were collected. The samples were prepared for label-free experiments using the SDT lysis method. An appropriate amount of SDT lysis buffer (4\% SDS, $100 \mathrm{mM}$ Tris-HCl, $\mathrm{pH}$ 7.6) was added to the human foetal frontal lobe tissues, and the samples were transferred to Lysing Matrix A tubes and homogenized twice using a FastPrep-24 homogenizer (MP Biomedicals) $(24 \times 2,6.0 \mathrm{~m} / \mathrm{s}, 60 \mathrm{~s})$ 
centrifuged at $14,000 \times \mathrm{g}$ for $15 \mathrm{~min}$. After centrifugation, the supernatants were collected and filtered through $0.22 \mu \mathrm{m}$ Spin-X centrifuge tube filters. The protein concentration was quantified using the BCA method ${ }^{2}$ (BCA Protein Assay Kit, Beyotime), and $20 \mu \mathrm{g}$ of each protein sample was subjected to $12 \%$ SDS-PAGE at $220 \mathrm{~V}$ for $40 \mathrm{~min}$. The protein extracts were mixed with 6x sample buffer (Beyotime, P0015F) and put into a boiling water bath for 5 min. Coomassie Brilliant Blue was used for staining.

\section{FASP digestion ${ }^{3}$}

DTT was added at a final concentration of $100 \mathrm{mM}$ to $200 \mu \mathrm{L}$ of each protein solution, and the samples were placed in a boiling water bath for $5 \mathrm{~min}$ and then cooled to room temperature. Then, $200 \mu \mathrm{L}$ of UA buffer ( $8 \mathrm{M}$ urea, $150 \mathrm{mM}$ Tris- $\mathrm{HCl}$ $\mathrm{pH}$ 8.5) was added, the samples were transferred to $30 \mathrm{kD}$ ultrafiltration centrifuge tubes and centrifuged at $12,500 \times \mathrm{g}$ for $15 \mathrm{~min}$, and the filtrates were discarded. Then, this process was repeated once. Next, $100 \mu \mathrm{L}$ IAA (100 mM IAA in UA) was added, and the samples were shaken at $600 \mathrm{rpm}$ for $1 \mathrm{~min}$, incubated for $30 \mathrm{~min}$ at room temperature in the dark and centrifuged at $12,500 \times \mathrm{g}$ for $15 \mathrm{~min}$. Then, $100 \mu \mathrm{L} \mathrm{UA}$ buffer was added, and the samples were centrifuged at $12,500 \times \mathrm{g}$ for $15 \mathrm{~min}$ at room temperature. This process was repeated twice. After that, the samples were treated $100 \mu \mathrm{L} 40 \mathrm{mM}$ NH4HCO3 solution and centrifuged at $12,500 \times \mathrm{g}$ for $15 \mathrm{~min}$ at room temperature, and this process was repeated 2 times. A $40 \mu \mathrm{L}$ aliquot of trypsin buffer (4 $\mu$ g trypsin in $40 \mu \mathrm{L} 40 \mathrm{mM}$ NH4HCO3 buffer) was added, and then the samples 
were shaken at $600 \mathrm{rpm}$ for $1 \mathrm{~min}$ and incubated at $37{ }^{\circ} \mathrm{C}$ for $16-18$ hours. Next, the samples were centrifuged at $12,500 \times \mathrm{g}$ for $15 \mathrm{~min}$, treated with $20 \mu \mathrm{L}$ of $40 \mathrm{mM}$ NH4HCO3 buffer, and centrifuged at $12,500 \times \mathrm{g}$ for $15 \mathrm{~min}$, and the filtrate was collected. The peptides were desalted with a C18 cartridge (Thermo Fisher Scientific), the peptide fragments were lyophilized and reconstituted, and the peptide concentration was quantified (OD280).

\section{$L C-M S / M S$ analysis}

The samples were separated by nanoflow liquid chromatography (Easy nLC, Thermo Fischer Scientific). Buffer A consisted of $0.1 \%$ formic acid in water, and buffer B consisted $0.1 \%$ formic acid in $80 \%$ acetonitrile. The chromatographic column was equilibrated at $100 \% \mathrm{~A}$, and LC separation was performed using a $50 \mu \mathrm{m} \mathrm{X} 15 \mathrm{~cm}$ Acclaim PepMap RSLC nano Viper column (Thermo Fisher Scientific) at a flow rate of $300 \mathrm{~nL} / \mathrm{min}$. The peptide fragments were separated by chromatography and analysed by a Q-Exactive Plus mass spectrometer (Thermo Fisher Scientific). The scanning range of the parent ions was $350-1,500 \mathrm{~m} / \mathrm{z}$, the resolution of first-order mass spectrometry was 70,000, the automatic gain control (AGC) target was 3e6, and the first-order maximum IT was $50 \mathrm{~ms}$. The mass-to-charge ratios of polypeptides and polypeptide fragments were obtained as follows. Twenty fragment patterns (MS2 scan, HCD) were collected after each full scan. The MS2 activation type was HCD, the isolation window was $2 \mathrm{~m} / \mathrm{z}$, the resolution of secondary mass spectrometry was 
17,500 , the microscan was 1 , the secondary maximum IT was $45 \mathrm{~ms}$, and the normalized collision energy was set to $27 \mathrm{eV}$.

\section{Protein identification and quantification}

570

Raw data were searched against the UniProt_HomoSapiens_20386_20180905

database. Protein identification and quantification were performed in MaxQuant ${ }^{4}$ version 1.5.5.1 (Max Planck Institute of Biochemistry) using the following parameters $^{5}$ : the enzyme was set to trypsin, two missed cleavages were allowed, the fixed modifications were set to carbamidomethyl $(\mathrm{C})$, the variable modifications were set to oxidation (M) and acetyl (Protein N-term), and the first and main search mass tolerances were set to 20 and 4.5 parts per million (ppm), respectively. A common contamination database was included to eliminate the effect of contamination proteins among the identified proteins. The identified peptides and proteins were filtered at a false discovery rate (FDR) of 0.01 . LFQ of proteins was performed using unique and razor peptides. The LFQ minimum ratio count was set to two.

\section{Screening of DEPs and AEPs}

To visualize the protein groups that were identified on only one side of the frontal lobe, an UpSetR diagram was created using the R package UpsetR v1.4.0 ${ }^{6}$.

Proteins that were detected in the bilateral frontal lobes in all samples were identified as the core protein set. The R package NormalyzerDE ${ }^{7}$ was used to normalize the abundance of the core proteins, and CycLoess normalization ${ }^{8}$ was applied to identify 
the DEPs between different GWs. The DEPs were identified by limma differential

589 expression analysis ${ }^{9}$ using a fold change $>2$ and FDR $<0.05$ as the criteria. A list of

590 the DEPs can be found in Supplementary file 2.

591 The protein groups that were detected in the bilateral frontal lobes at each GW were

592 used for AEP screening. Proteins with a fold change in LFQ intensity $>2$ and $p$ value

$593<0.05$ between the left and right frontal lobes were considered to be asymmetrically

594 expressed. The fold change data for the AEPs were used to generate a heatmap using

595 TBtools software ${ }^{10}$.

\section{PPI network analysis}

PPI networks are of great significance for revealing the interactions between proteins.

In this study, the online tool Search Tool for the Retrieval of Interacting

Genes/Proteins (STRING) (http://string-db.org) was used.

601

\section{Enrichment analysis, UniProt keyword annotation and functional annotation}

603

604

605

606

607

608

\section{clustering}

GO enrichment analysis and GSEA were performed with the R package

'ClusterProfiler'"11 and Bioconductor annotation package 'org.Hs.eg.db'. The

'GOplot' package was used to visualize the distribution of asymmetrically expressed proteins enriched in certain GO terms ${ }^{11}$. The $\mathrm{C} 2$ canonical pathway database, the $\mathrm{C} 5$ GO, ZHONG PFC gene set ${ }^{13}$, the MANNO MIDBRAIN NEUROTYPES gene set ${ }^{14}$ 
and the FAN EMBRYONIC CTX gene set ${ }^{15}$, which are available through the

610 Molecular Signatures Database (v.7.4) ${ }^{16}$ were used for GSEA. UniProt keyword

611 annotation was performed using UniProt Retrieve/ID mapping

612 (https://www.uniprot.org/). Functional annotation clustering was carried out using

DAVID (https://david.ncifcrf.gov/).

614

615

\section{STEM analysis}

616 In the current study, we used STEM software ${ }^{17}$ to study the change in the expression

617 pattern of core proteins in the bilateral frontal lobes with age. The normalized LFQ

618 values of the core proteins were subjected to downstream clustering analysis based

619 using the default settings. Clusters with a $p$ value $\leq 0.05$ were considered statistically

620 significant.

621

622

\section{Correlation analysis and PCA}

623 Correlation analysis and PCA were carried out with the cor and prcomp functions,

624 respectively, of R using the correlation matrix of the CycLoess normalized core

625 protein data.

626 


\section{References}

1. Zhu, Y. et al. Proteomic Analysis of Solid Pseudopapillary Tumor of the Pancreas Reveals Dysfunction of the Endoplasmic Reticulum Protein Processing Pathway. Mol. Cell. Proteomics. 13, 2593-2603 (2014).

2. Smith, P. K. et al. Measurement of protein using bicinchoninic acid. Anal. Biochem. 150, 76-85 (1985).

3. Wisniewski, J. R., Zougman, A., Nagaraj, N. \& Mann, M. Universal sample preparation method for proteome analysis. Nat. Methods. 6, 359-362 (2009).

4. Cox, J. \& Mann, M. MaxQuant enables high peptide identification rates, individualized p.p.b.-range mass accuracies and proteome-wide protein quantification. Nat. Biotechnol. 26, 1367-1372 (2008).

5. Tyanova, S., Temu, T. \& Cox, J. The MaxQuant computational platform for mass spectrometrybased shotgun proteomics. Nat. Protoc. 11, 2301-2319 (2016).

6. Lex, A., Gehlenborg, N., Strobelt, H., Vuillemot, R. \& Pfister, H. UpSet: Visualization of Intersecting Sets. IEEE T. Vis. Comput. Gr. 20, 1983-1992 (2014).

7. Willforss, J., Chawade, A. \& Levander, F. NormalyzerDE: Online Tool for Improved Normalization of Omics Expression Data and High-Sensitivity Differential Expression Analysis. $J$. Proteome Res. 18, 732-740 (2019).

8. Gentleman, R. Bioinformatics and computational biology solutions using $R$ and Bioconductor. (Springer Science+Business Media, New York, 2005).

9. Ritchie, M. E. et al. limma powers differential expression analyses for RNA-sequencing and microarray studies. Nucleic Acids Res. 43, e47 (2015).

10. Chen, C. et al. TBtools: An Integrative Toolkit Developed for Interactive Analyses of Big Biological Data. Mol. Plant. 13, 1194-1202 (2020).

11. Wu, T. et al. clusterProfiler 4.0: A universal enrichment tool for interpreting omics data. Innovation (N Y). 2, 100141 (2021).

12. Walter, W., Sanchez-Cabo, F. \& Ricote, M. GOplot: an R package for visually combining expression data with functional analysis. Bioinformatics. 31, 2912-2914 (2015).

13. Zhong, S. et al. A single-cell RNA-seq survey of the developmental landscape of the human prefrontal cortex. Nature. 555, 524-528 (2018).

14. La Manno, G. et al. Molecular Diversity of Midbrain Development in Mouse, Human, and Stem Cells. Cell. 167, 566-580 (2016).

15. Fan, X. et al. Spatial transcriptomic survey of human embryonic cerebral cortex by single-cell RNAseq analysis. Cell Res. 28, 730-745 (2018).

16. Liberzon, A. et al. Molecular signatures database (MSigDB) 3.0. Bioinformatics. 27, 1739-1740 (2011).

17. Ernst, J. \& Bar-Joseph, Z. STEM: a tool for the analysis of short time series gene expression data. BMC Bioinformatics. 7, 191 (2006). 
668 Author contributions

669

670 Competing interest

671 The authors declare that they have no competing interests.

672 
Fig. 1. Proteins identified in the foetal frontal lobes at different GWs

675

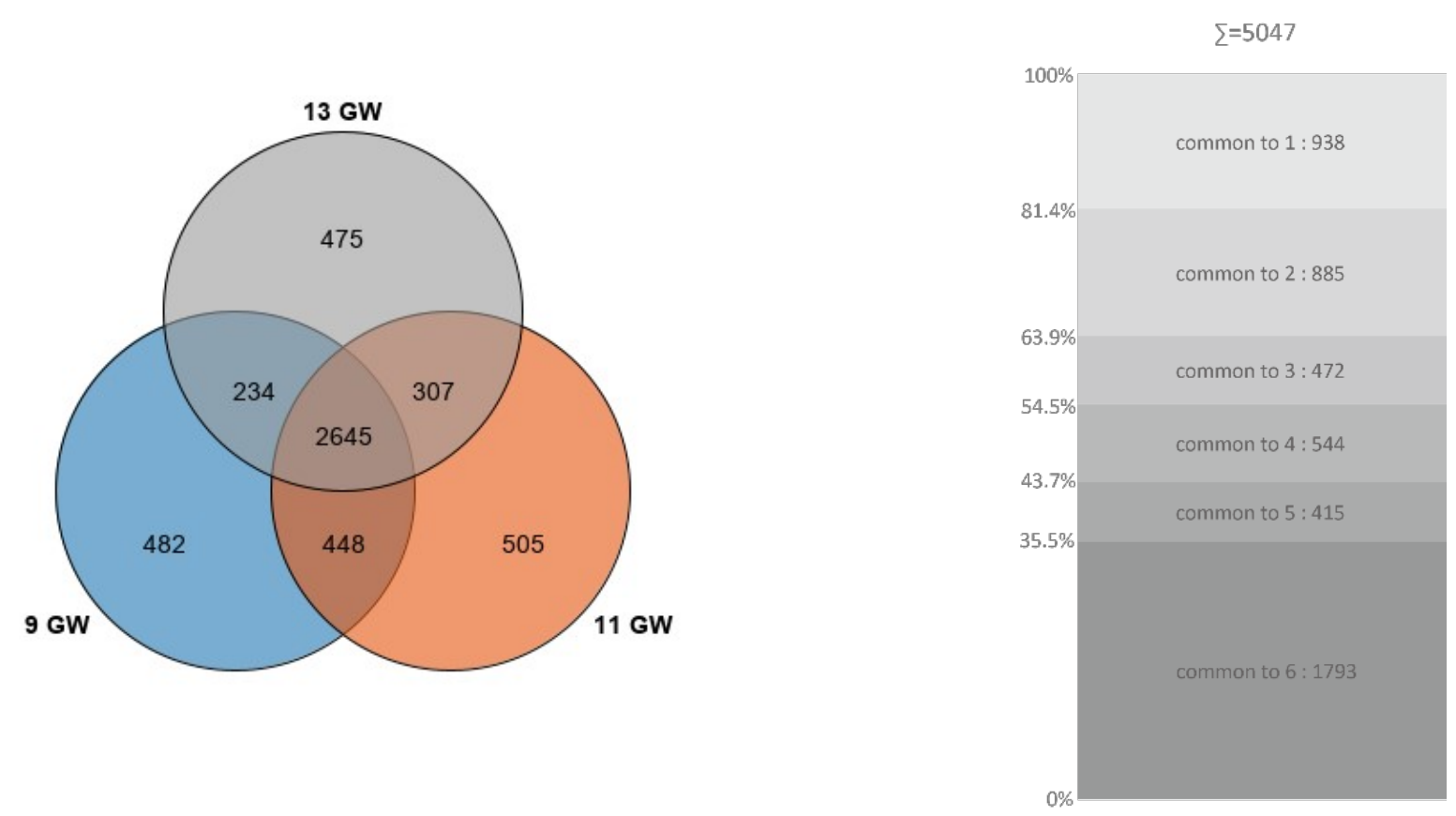

676 a The protein set identified in all three samples contained 2645 proteins, and the core

677 protein set identified in all six tissues contained 1973 proteins.

678 

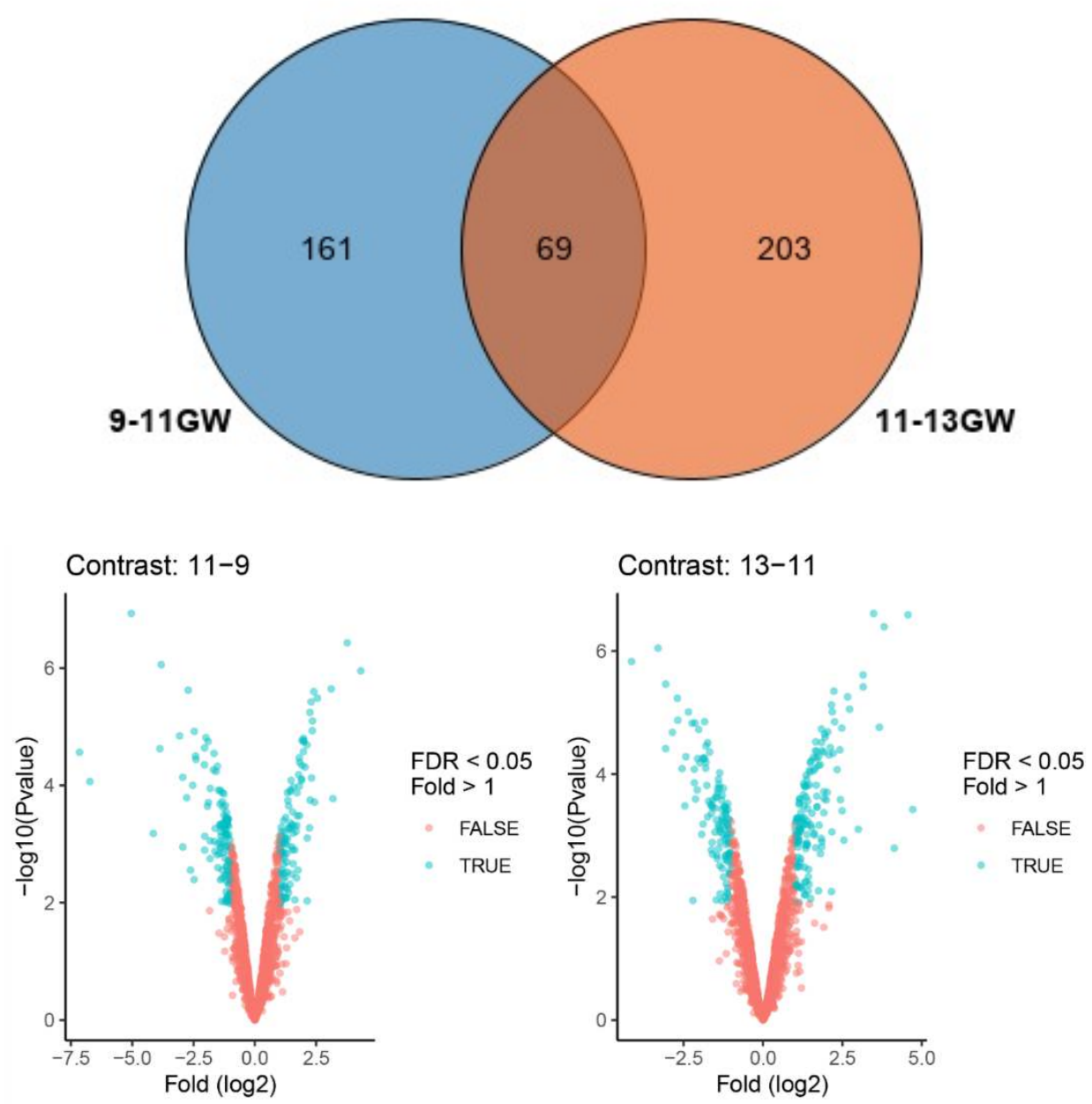

679 b Volcano map of core proteins that showed significant differences in abundance

680 between GWs 9-11 and GWs $11-13($ FDR $<0.05$, $\log 2$ Fold $>1)$. For a complete list of

681 DEPs, see Supplementary Table 1. Sixty-nine core proteins were shared between GWs

682 9-11 and GWs 11-13. Detailed information on the protein names can be found in

683 Supplementary Table 3.

684 


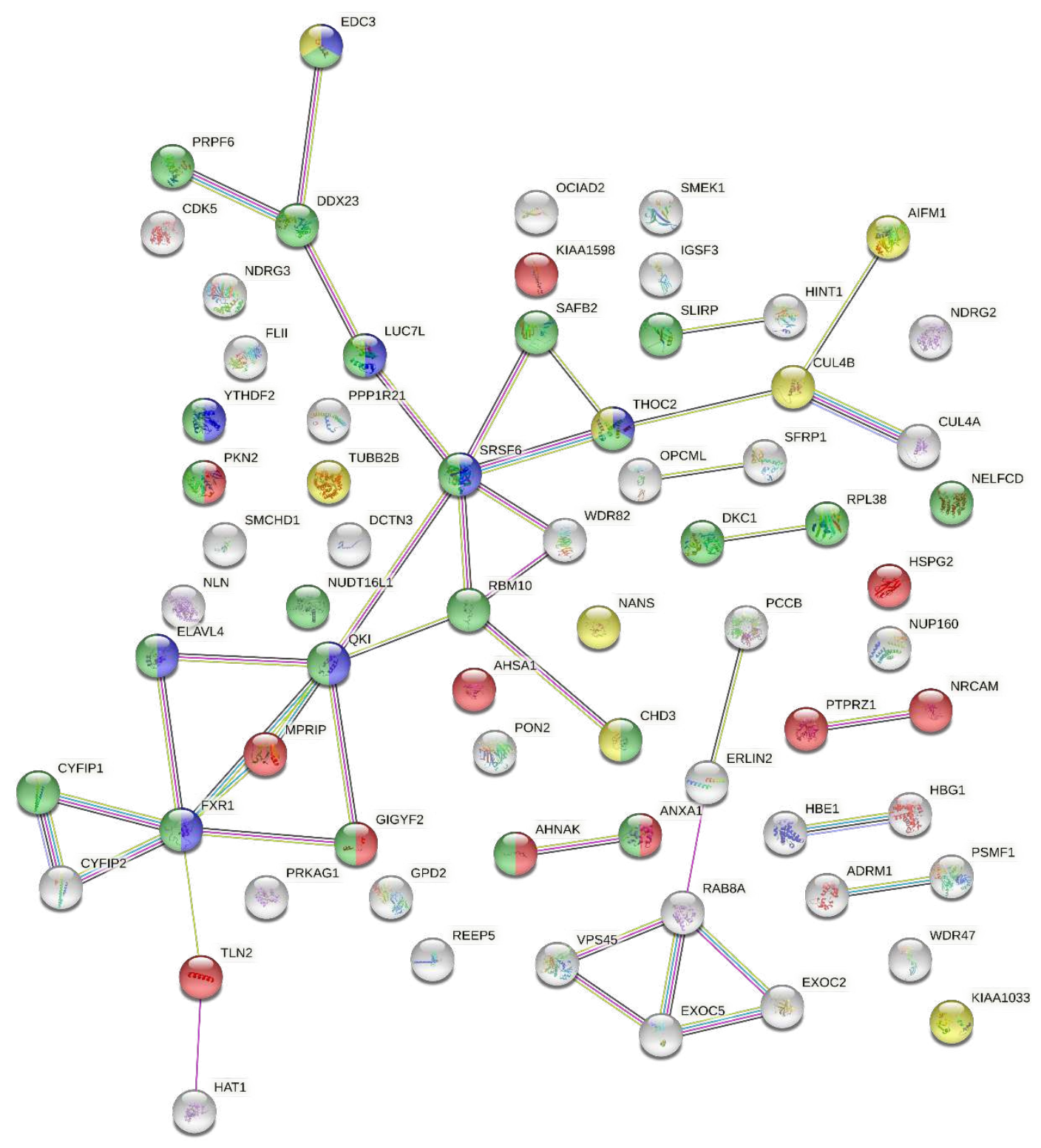

686 c Visualization of the PPI network of core proteins shared between GWs 9-11 and

687 GWs 11-13 (PPI enrichment p value: 0.0057). The nodes represent proteins, and

688 protein groups enriched in different functions are indicated by the different colours;

689 protein groups enriched in mRNA binding are purple (count $=8, \mathrm{FDR}=0.016$ ), those

690 enriched in cell adhesion molecule binding are red (count $=11, \mathrm{FDR}=0.0081$ ), those

691 enriched RNA binding are green (count $=23, \mathrm{FDR}=2.88 \mathrm{e}-05)$ and those enriched in 
693 predicted interactions.

694

$695 \quad$ Fig. 1

696 


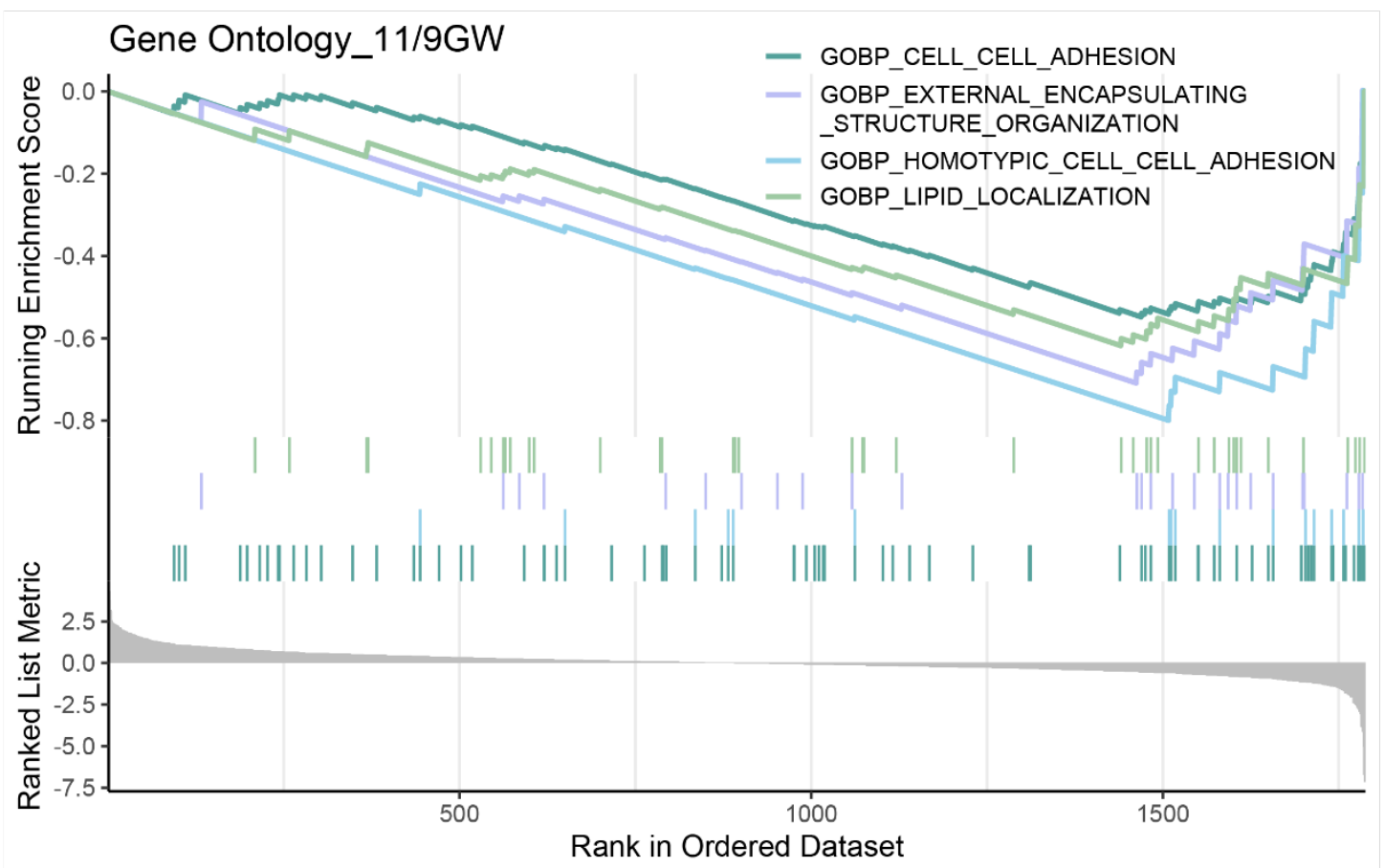

698

699 a GSEA showed that the DEPs between the GW 11 samples and GW 9 samples were

700 significantly enriched in the biological processes cell-cell adhesion, organization of

701 external encapsulating structure, and lipid localization. 


\section{Gene Ontology_13/11GW}

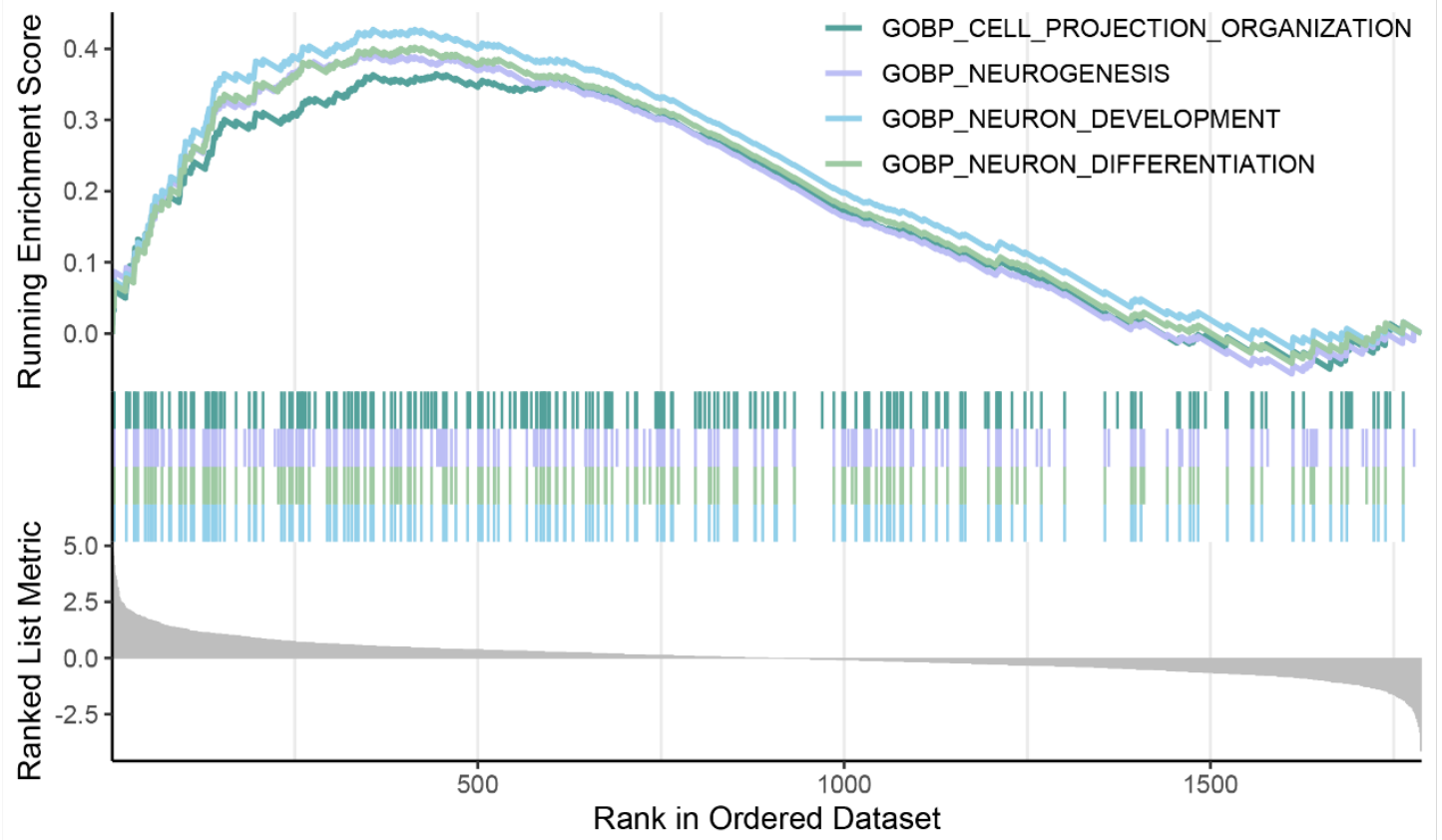

704 b The DEPs between the GW 13 samples and the GW 11 samples were significantly

705 enriched in the biological processes cell projection organization, neurogenesis,

706 neuronal development and neuronal differentiation processes. 


\section{DNA REPLICATION_13/9GW}

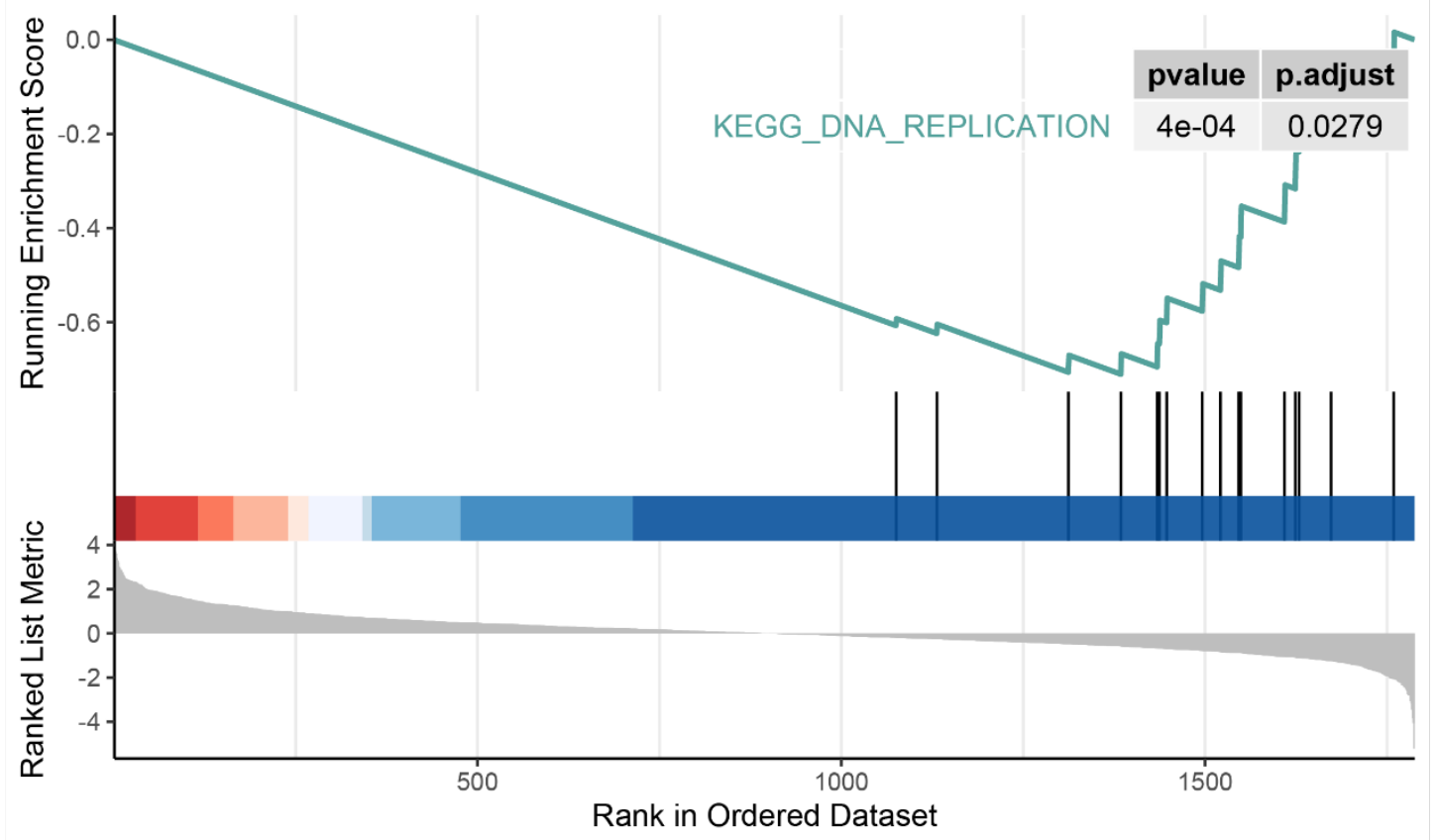

708

709 c The DEPs between the GW 13 samples and GW 9 samples were enriched in DNA

710 replication pathways. 
Cell type signature_11/9GW

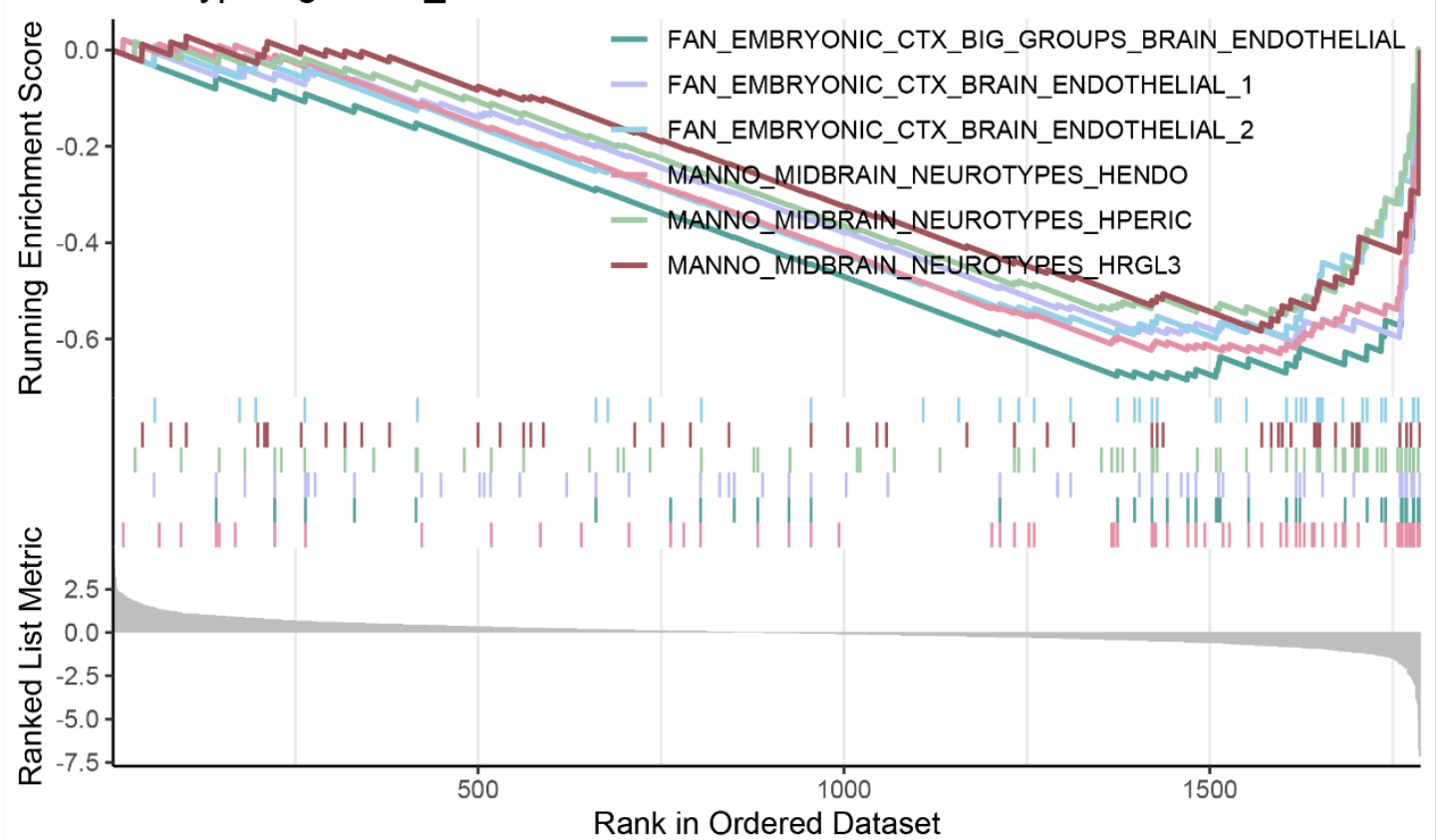

Cell type signature_13/11GW

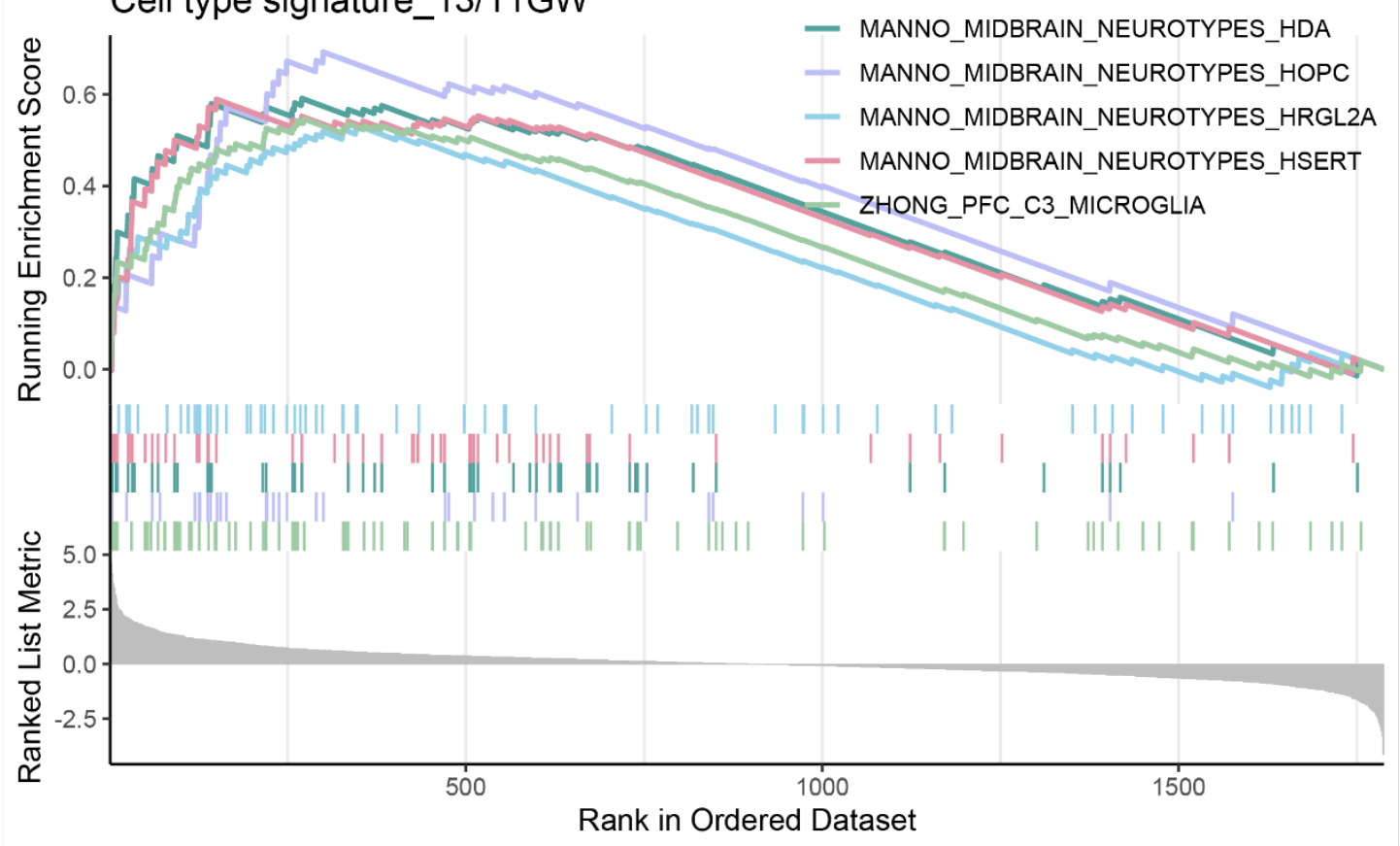

d GSEA of cell type signatures in GW 11 vs. GW 9 samples (top) and GW 13 vs. GW

71311 samples (bottom). CTX, cortex; HENDO, human endothelial cell; HPERIC,

714 human pericyts; HRGL3, human radial glia-like cell 3; HDA, human dopaminergic

715 neuron; HOPC, human oligodendrocyte precursor cell; HRGL2A, human radial glia-

716 like cell 2A; HSERT, human serotonergic neuron; PFC, prefrontal cortex. 
$718 \quad$ Fig. 2

719 

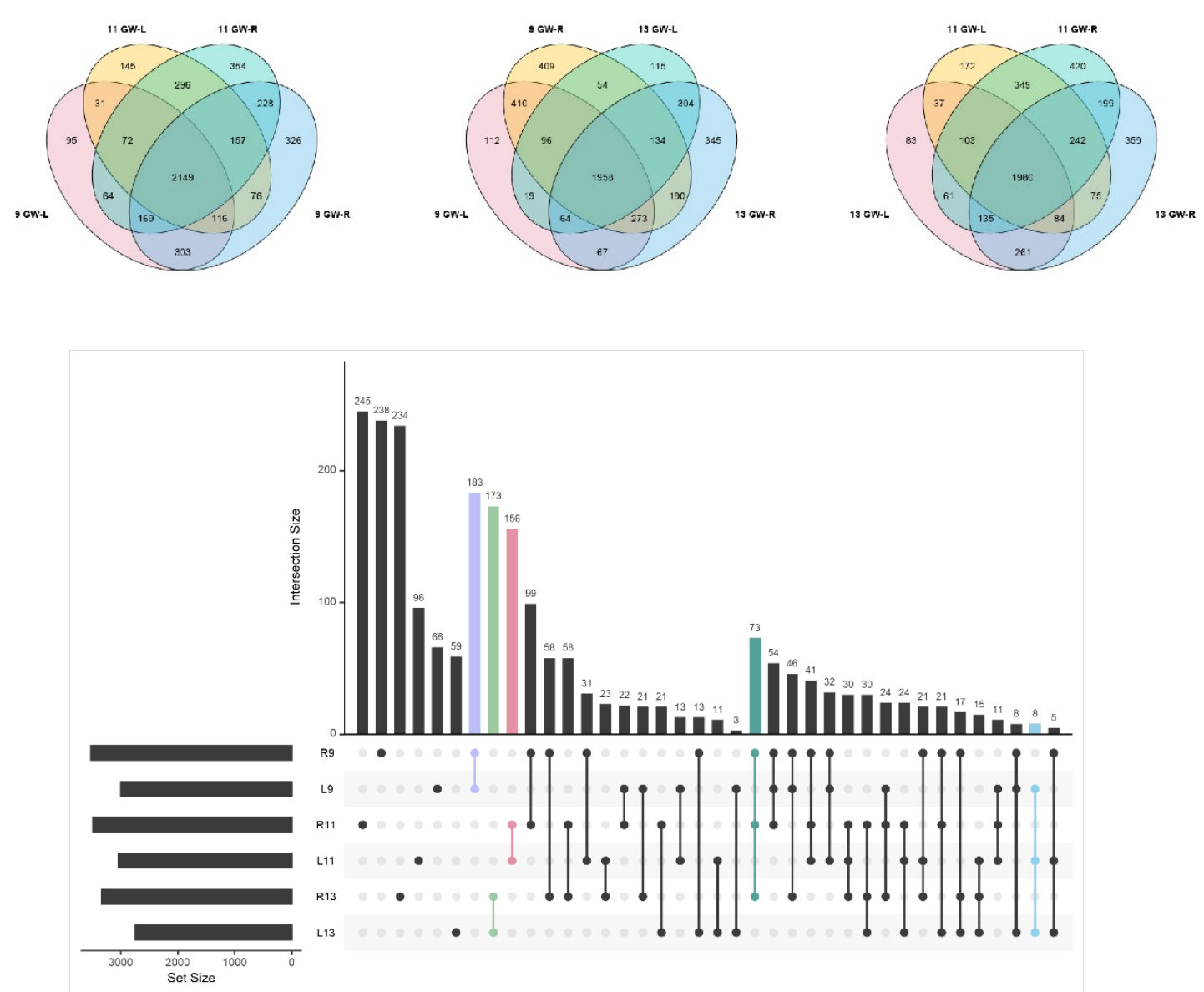

722 a Overlapping proteins in the bilateral frontal lobes at different GWs. An UpSetR

723 diagram was created to visualize the proteins identified in only one side of the frontal

724 lobe ( 8 on the left side and 73 on the right side). 


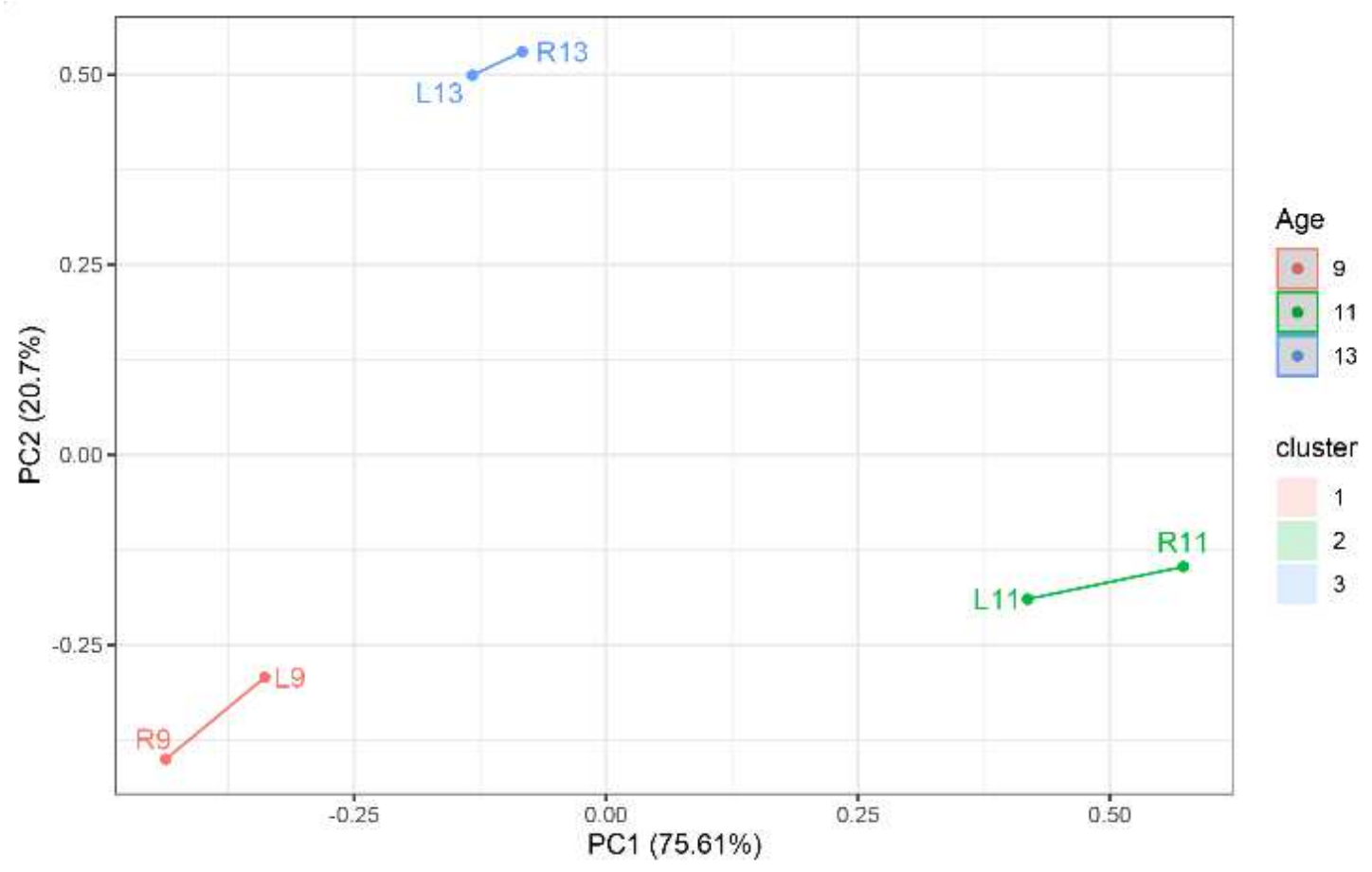

725

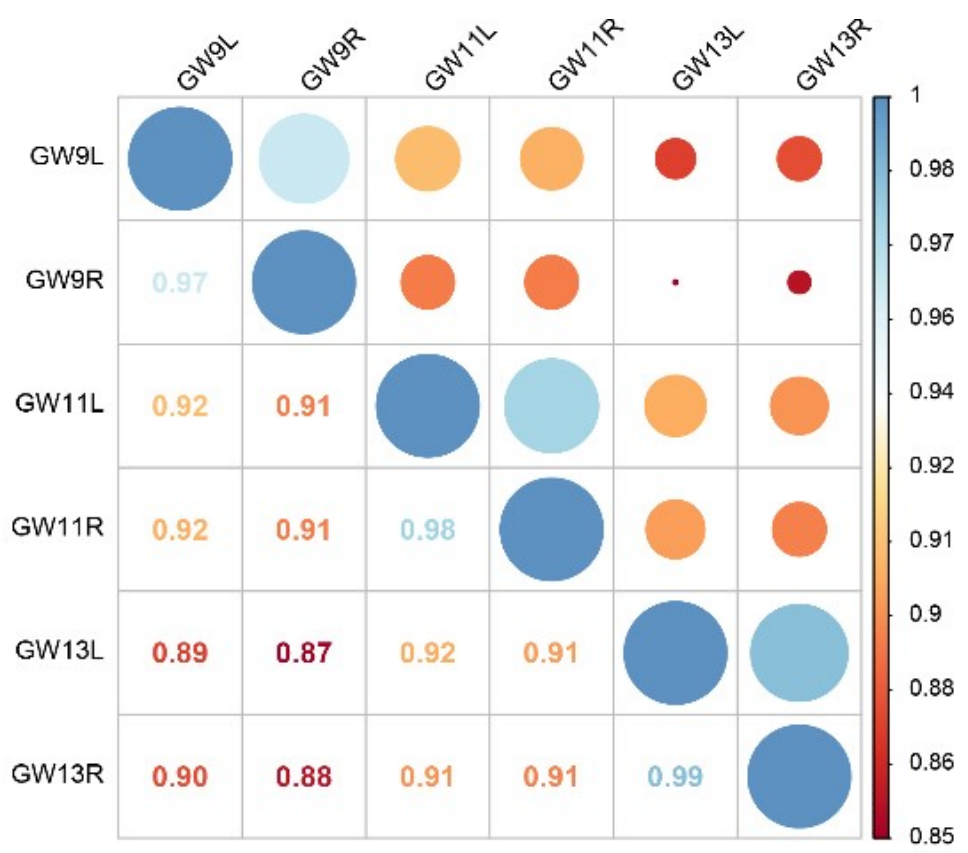

b PCA (top) and Pearson correlation analysis (bottom) of samples from different

727 GWs. 


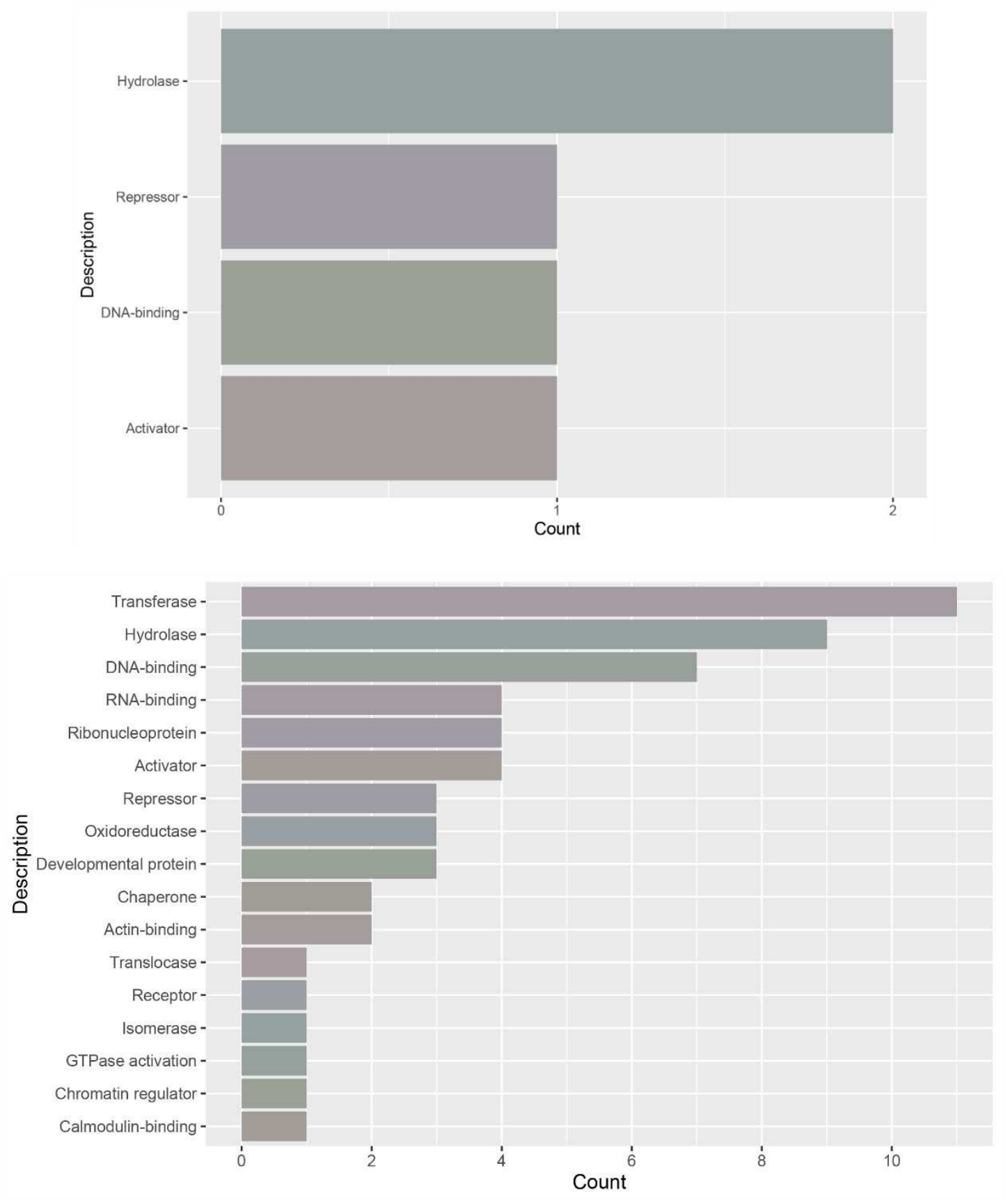

729 c Molecular functions associated with both the proteins expressed only on the left side

730 (top) and those expressed only on the right side (bottom), as identified by a search of

731 the UniProtKB database. 


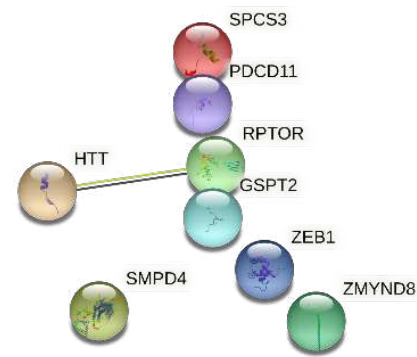

734 d Visualization of the PPI network of proteins expressed only on the left side (PPI 735 enrichment $p$ value: 0.535 ). 


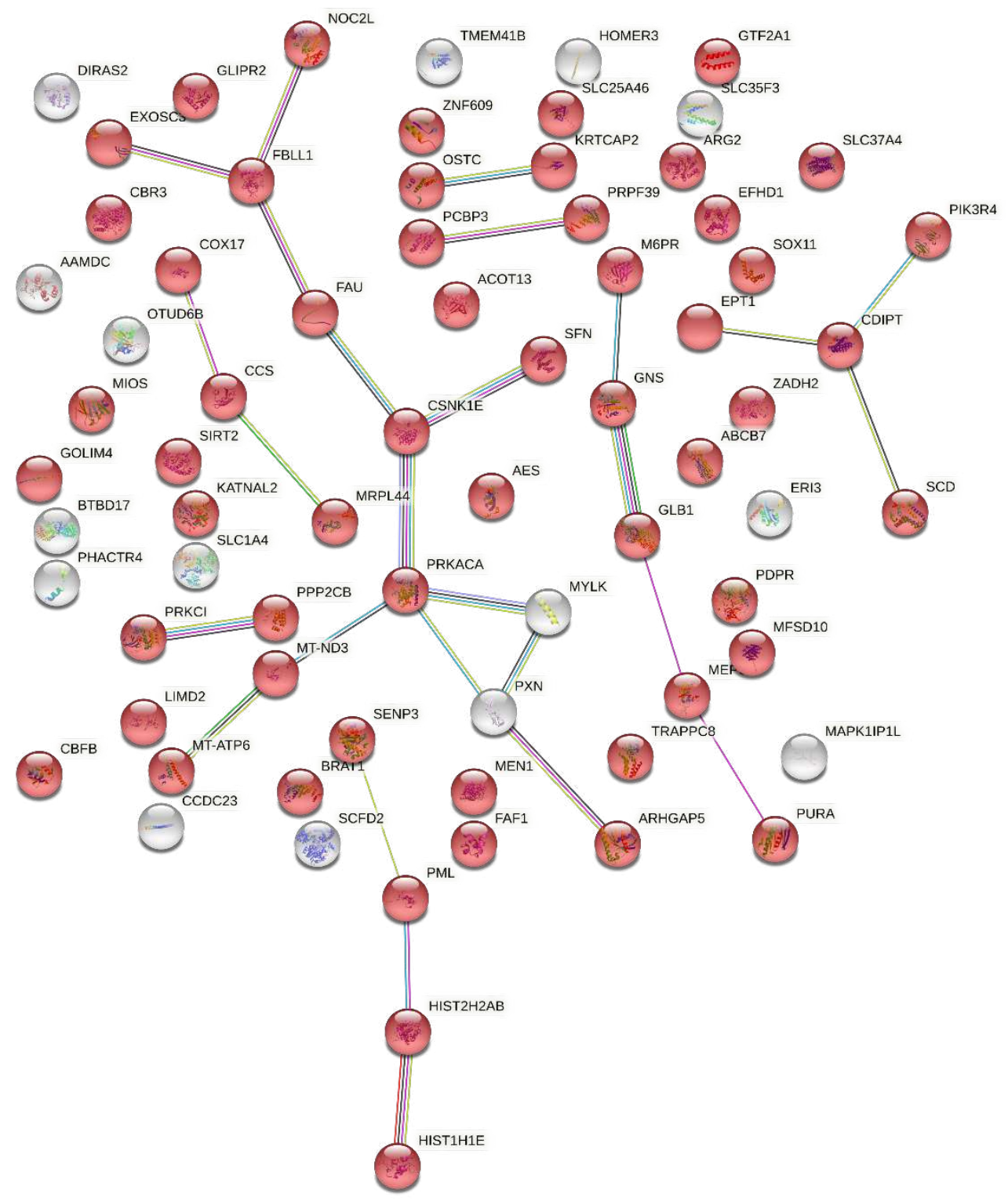

738 e Visualization of the PPI network of proteins expressed only on the right side (PPI

739 enrichment $p$ value: 0.396 ). Proteins enriched in the molecular function intracellular

740 membrane-bound organelle are marked in red $(\mathrm{FDR}=0.042$, count $=57)$.

Fig. 3 

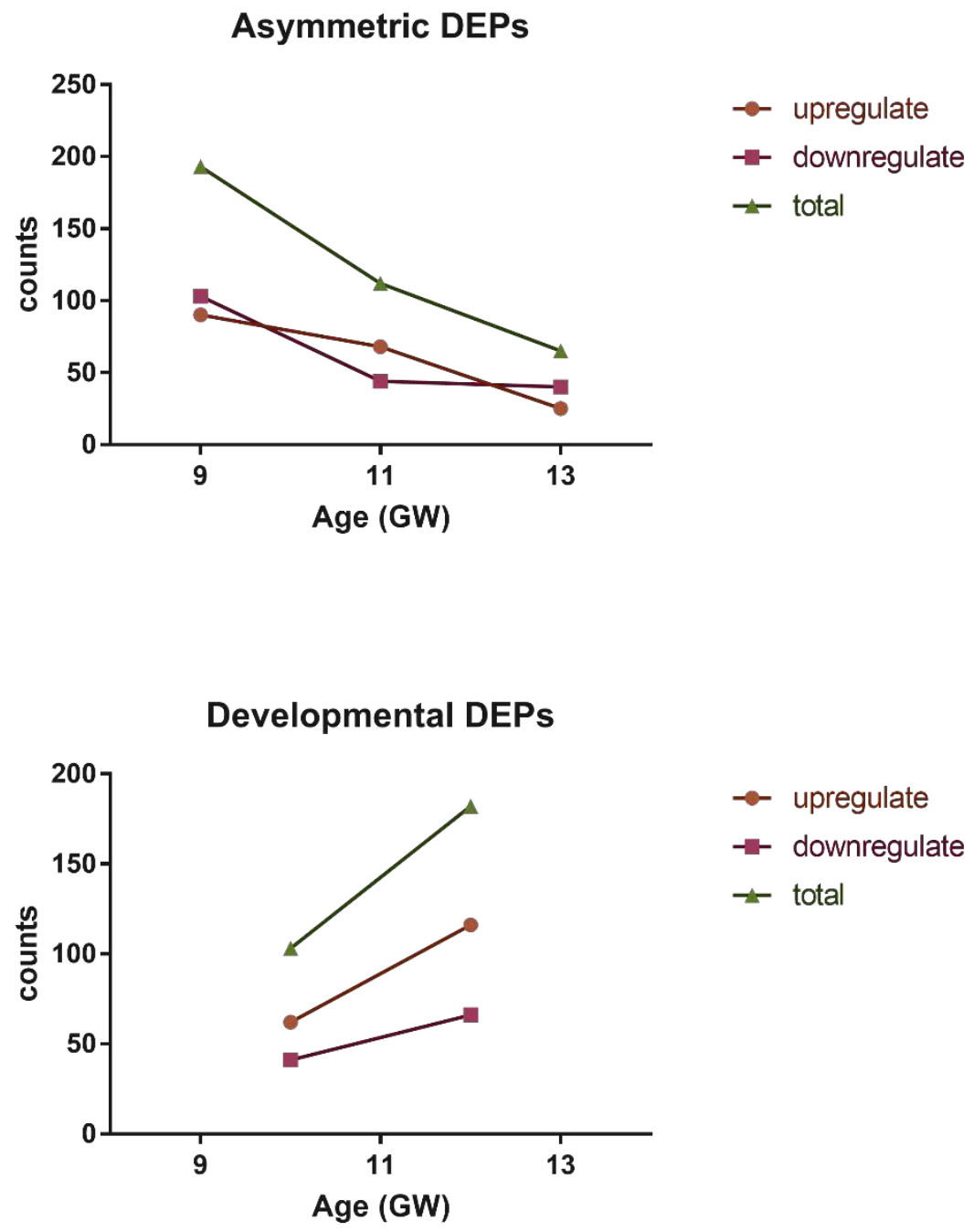

745 a The number of DEPs increased while the number of AEPs decreased from GW 11 to

746 GW 13. 


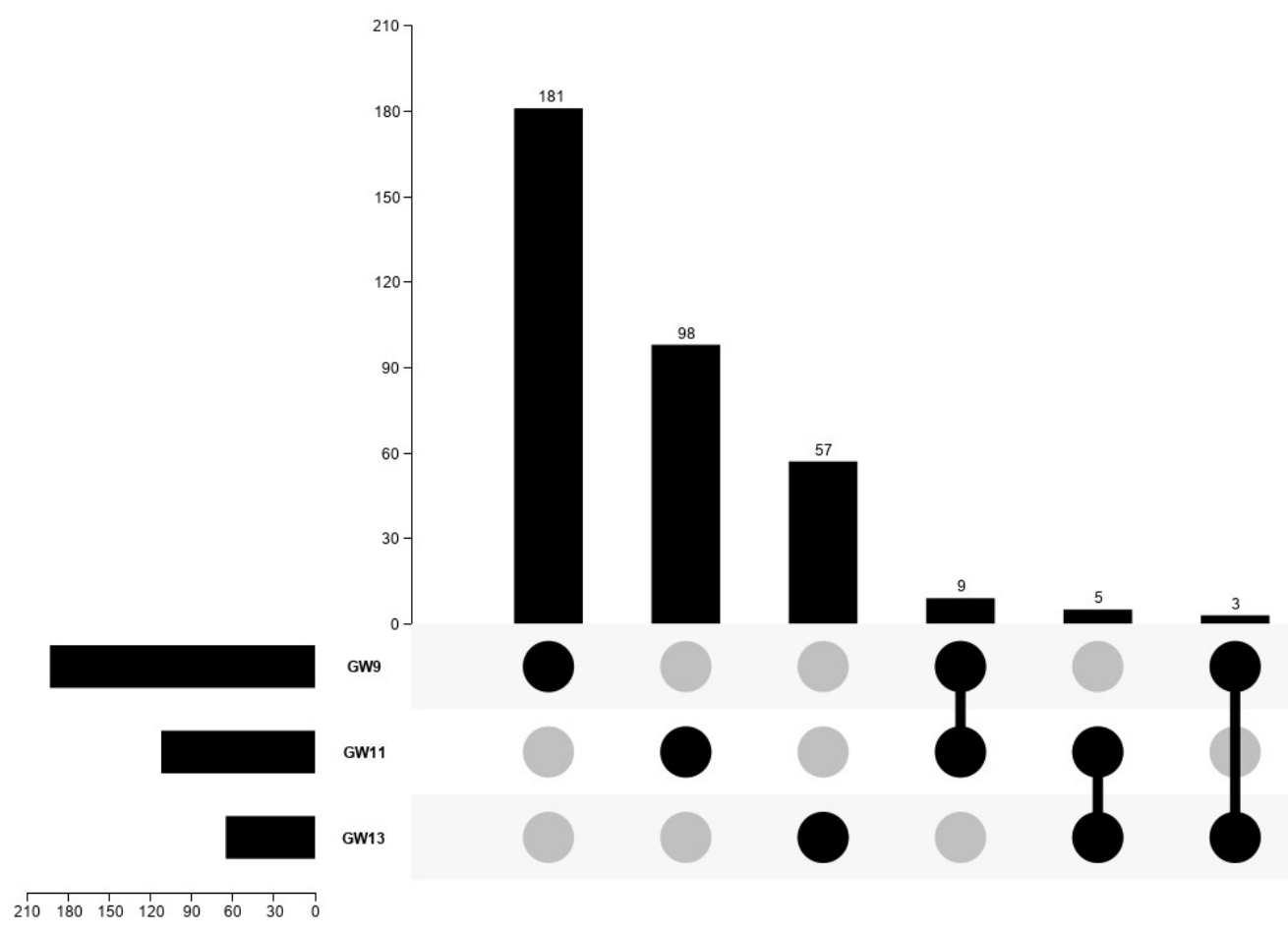

b Seventeen $(9+5+3)$ core AEPs were shared between two different GWs. 


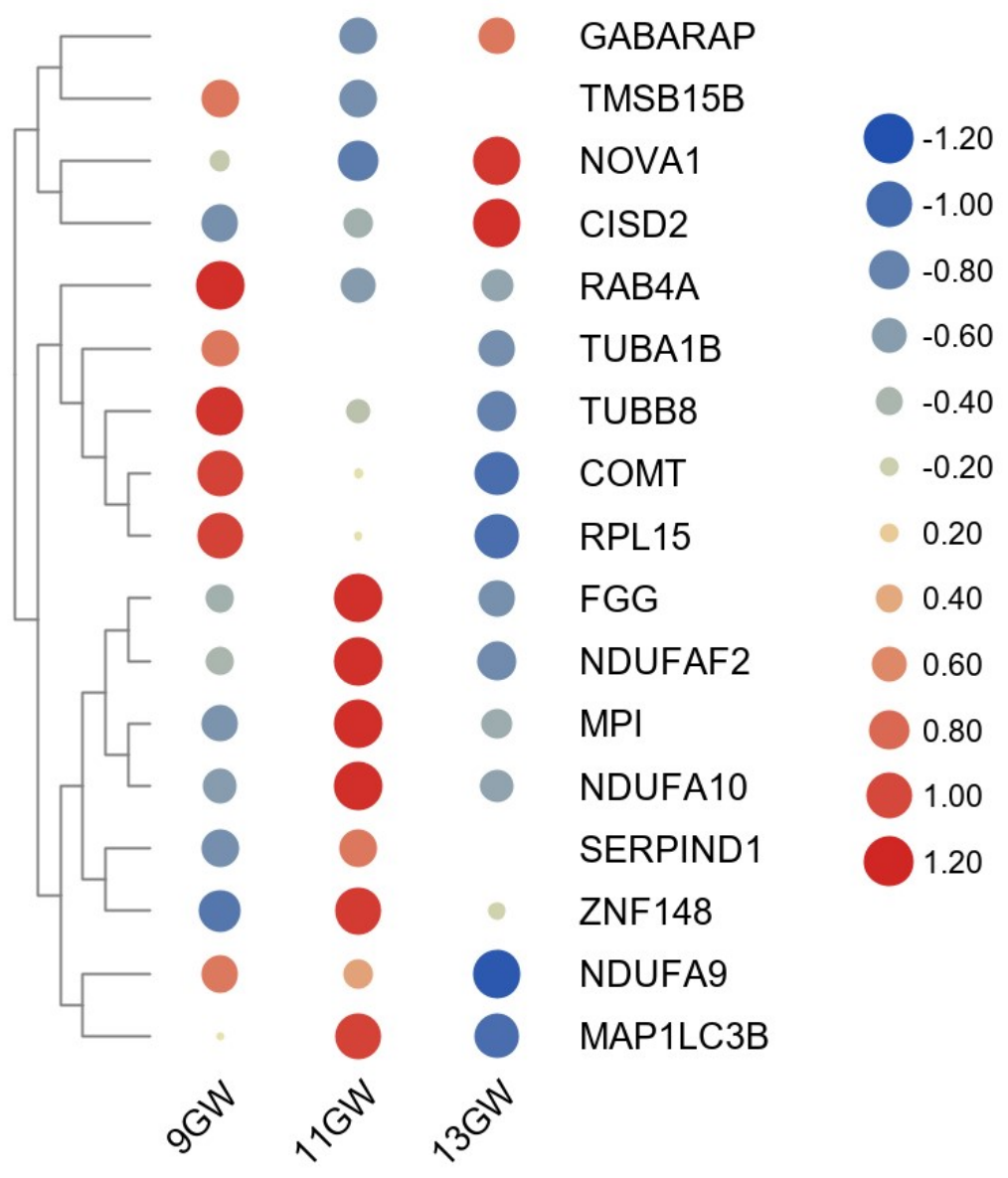

750 c Heatmap showing the expression changes of core AEPs at the three GWs.

751 


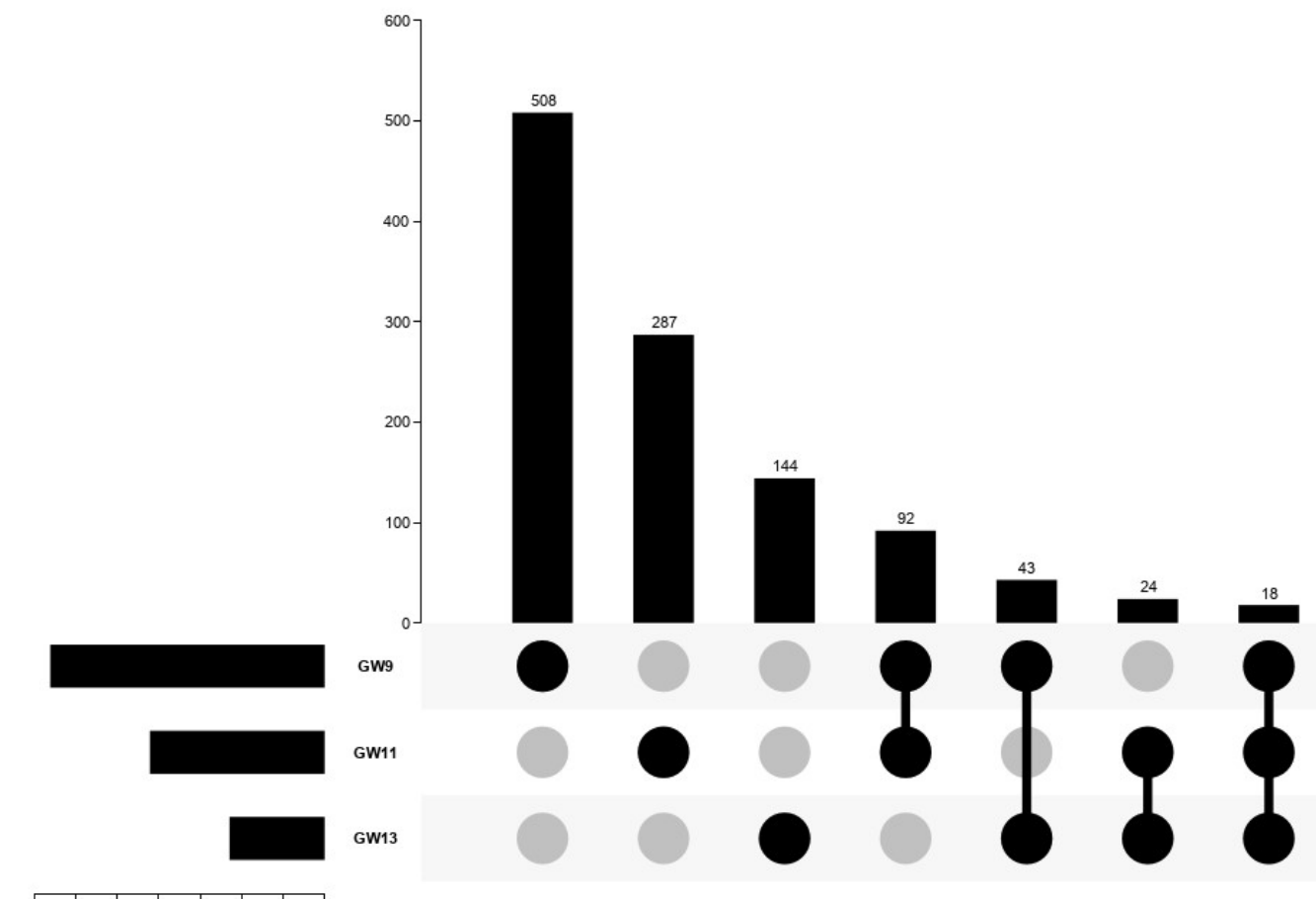

$700 \quad \begin{array}{lllllll}1 & 1 & 1 & 1 & 1 & 1 & 1 \\ 700 & 400 & 300 & 200 & 100 & 0\end{array}$

d Eighteen AEPs were shared between all three GWs. 


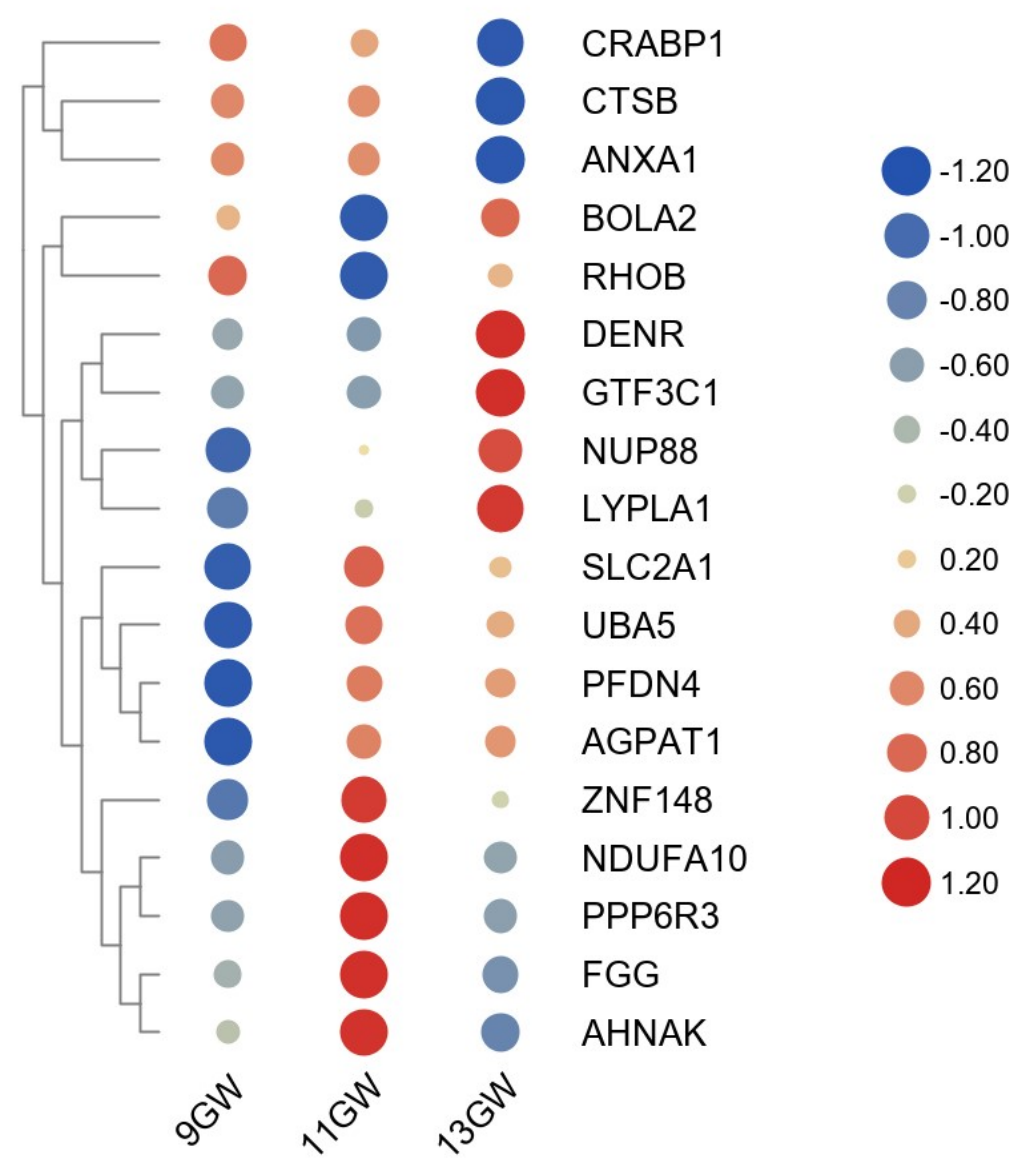

754 e Heatmap showing the expression changes in the 18 AEPs shared between all three 755 GWs.

756

$757 \quad$ Fig. 4 
Fig. 5. GO enrichment analysis and GSEA of AEPs
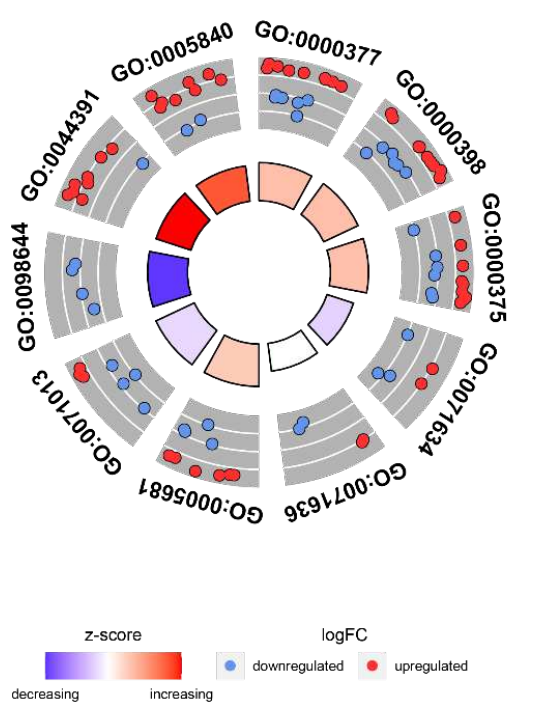

760

a
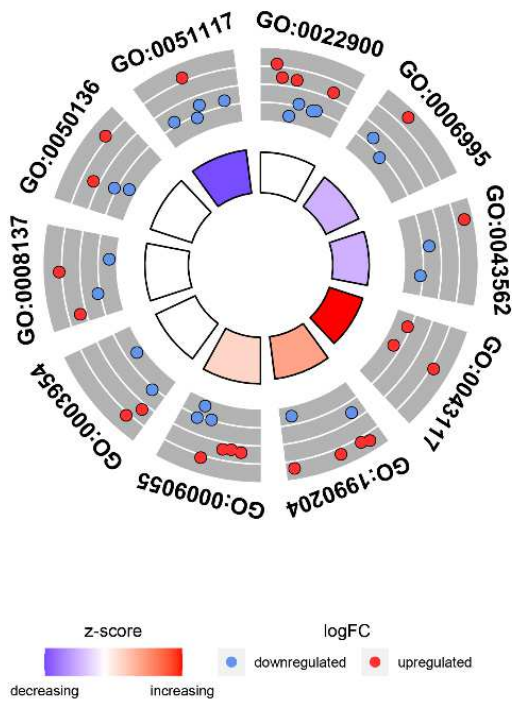

761

b

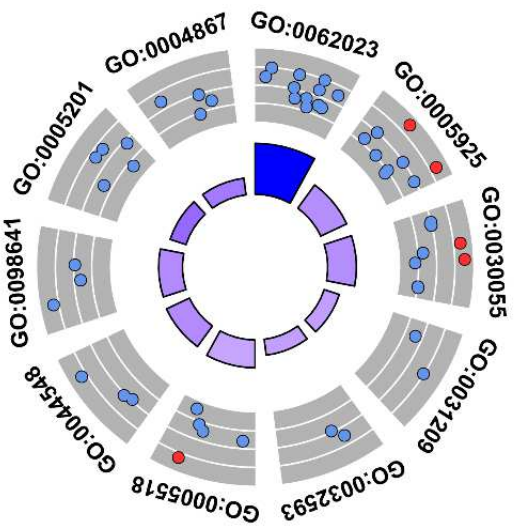

\begin{tabular}{|c|c|}
\hline ID & Description \\
\hline GO:0000377 & $\begin{array}{l}\text { RNA splicing, via transesterification reactions } \\
\text { with bulged adenosine as nucleophile }\end{array}$ \\
\hline GO:0000398 & mRNA splicing, via spliceosome \\
\hline GO:0000375 & RNA splicing, via transesterification reactions \\
\hline GO:0071634 & regulation of transforming growth factor beta production \\
\hline GO:0071636 & positive regulation of transforming growth factor beta production \\
\hline GO:0005681 & spliceosomal complex \\
\hline GO:0071013 & catalytic step 2 spliceosome \\
\hline GO:0098644 & complex of collagen trimers \\
\hline GO:0044391 & ribosomal subunit \\
\hline GO:0005840 & ribosome \\
\hline
\end{tabular}

\begin{tabular}{|c|c|}
\hline ID & Description \\
\hline GO:0022900 & electron transport chain \\
\hline GO:0006995 & cellular response to nitrogen starvation \\
\hline GO:0043562 & cellular response to nitrogen levels \\
\hline GO:0043117 & positive regulation of vascular permeability \\
\hline GO:1990204 & oxidoreductase complex \\
\hline GO:0009055 & electron transfer activity \\
\hline GO:0003954 & NADH dehydrogenase activity \\
\hline GO:0008137 & NADH dehydrogenase (ubiquinone) activity \\
\hline GO:0050136 & NADH dehydrogenase (quinone) activity \\
\hline GO:0051117 & ATPase binding \\
\hline
\end{tabular}

\begin{tabular}{|c|c|}
\hline ID & $\begin{array}{c}\text { Description } \\
\text { GO:0062023 }\end{array}$ \\
\hline GO:0005925 & collagen-containing extracellular matrix \\
\hline GO:0030055 & focal adhesion \\
\hline GO:0031209 & SCARstrate junction \\
\hline GO:0032593 & insulin-responsive compartment \\
\hline GO:0005518 & collagen binding \\
\hline GO:0044548 & S100 protein binding \\
\hline GO:0098641 & cadherin binding involved in cell-cell adhesion \\
\hline GO:0005201 & extracellular matrix structural constituent \\
\hline GO:0004867 & serine-type endopeptidase inhibitor activity \\
\hline
\end{tabular}

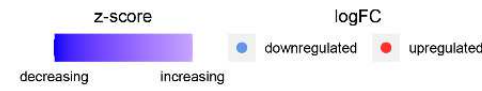


763 GO terms enriched from AEPs at GW 9 (a), GW 11 (b) and GW 13 (c). The blue dots

764 represent proteins that were highly expressed on the right side, and the red dots

765 represent proteins that were highly expressed on the left side. For a complete list of

766 enriched GO terms, see Supplement Tables 14-16.

767 


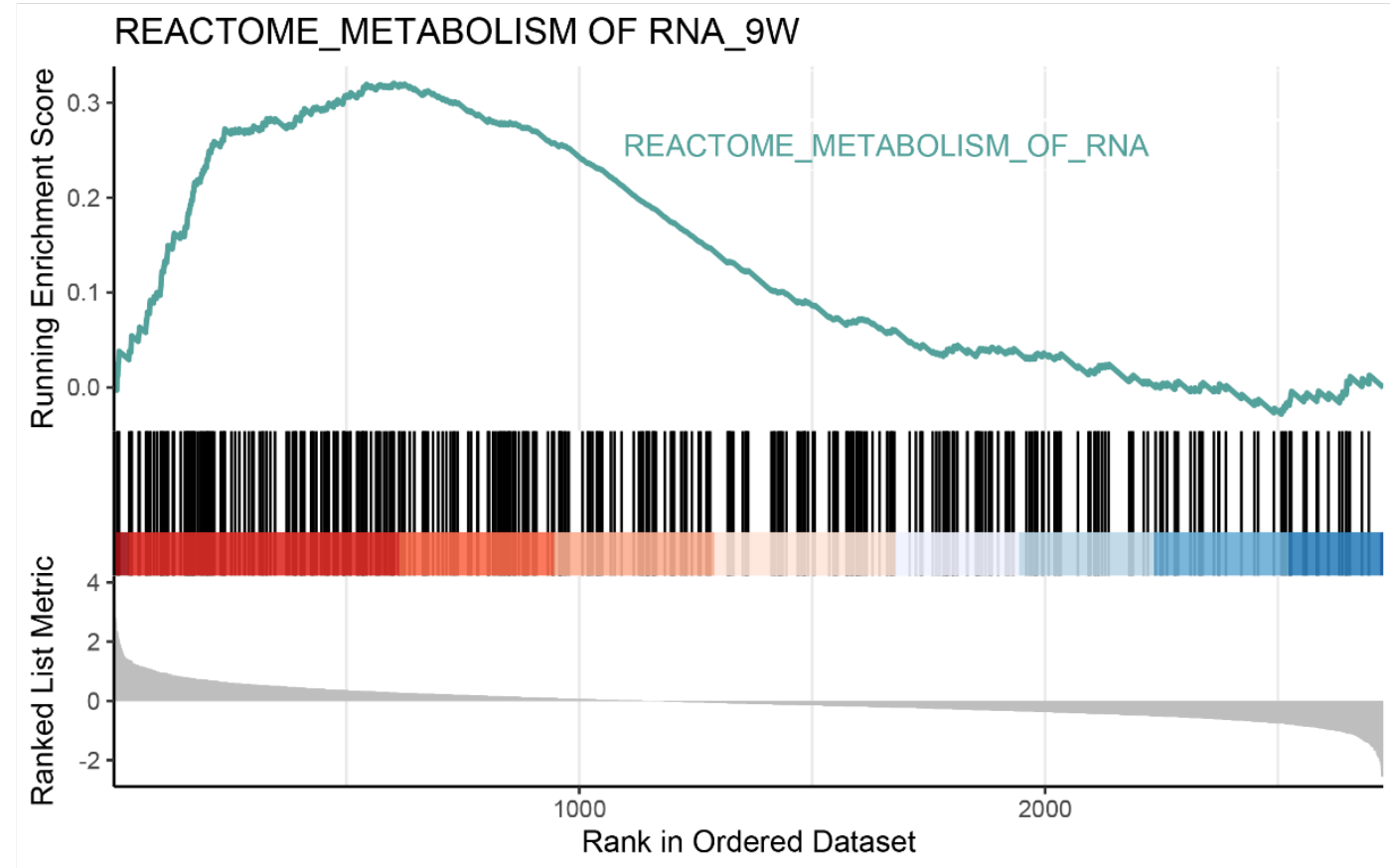

769 d GSEA of proteins that were highly expressed on the left side at GW 9. The analysis

770 showed that RNA metabolism was significantly associated with many proteins

771 expressed only in the left frontal (NES $=0.32)$. 


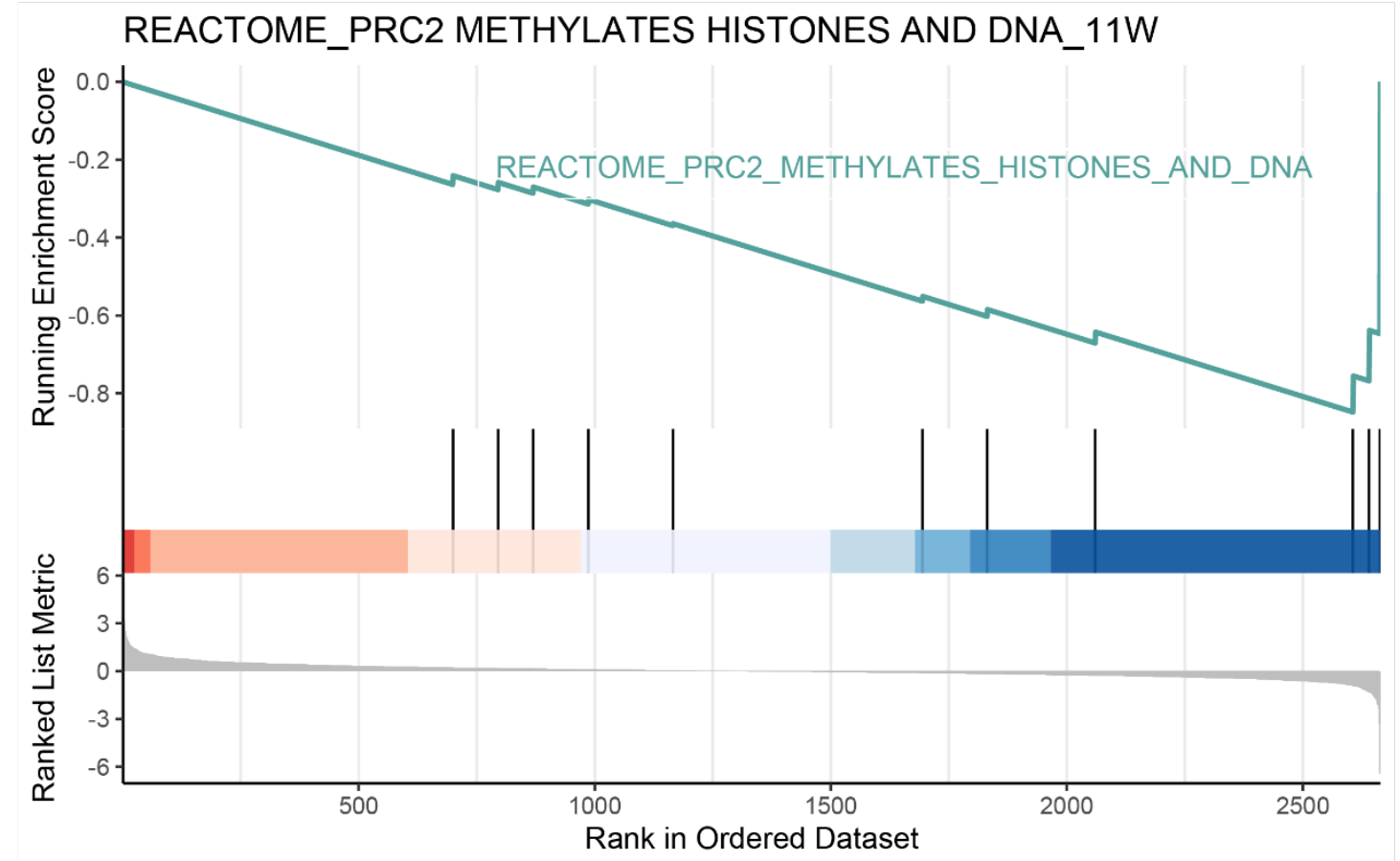

773 e GSEA of proteins that were highly expressed on the right side at GW 11. The

774 analysis showed that PRC2-mediated methylation of histones and DNA was the

775 pathway that was significantly associated with many of the proteins expressed in the

776 right frontal lobe $(\mathrm{NES}=-0.85)$. 


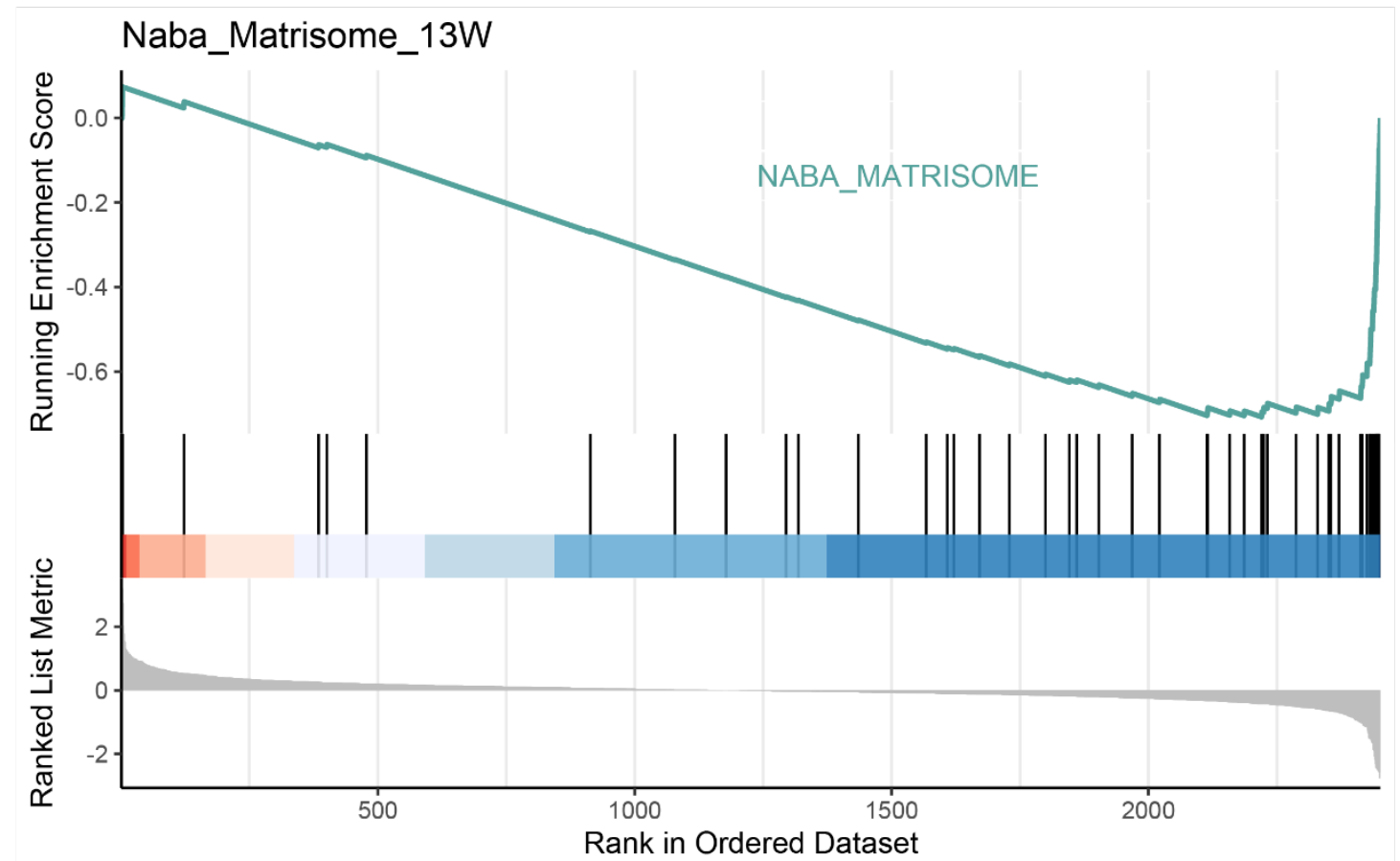

ZHONG_PFC_C8 ORG PROLIFERATING_13W

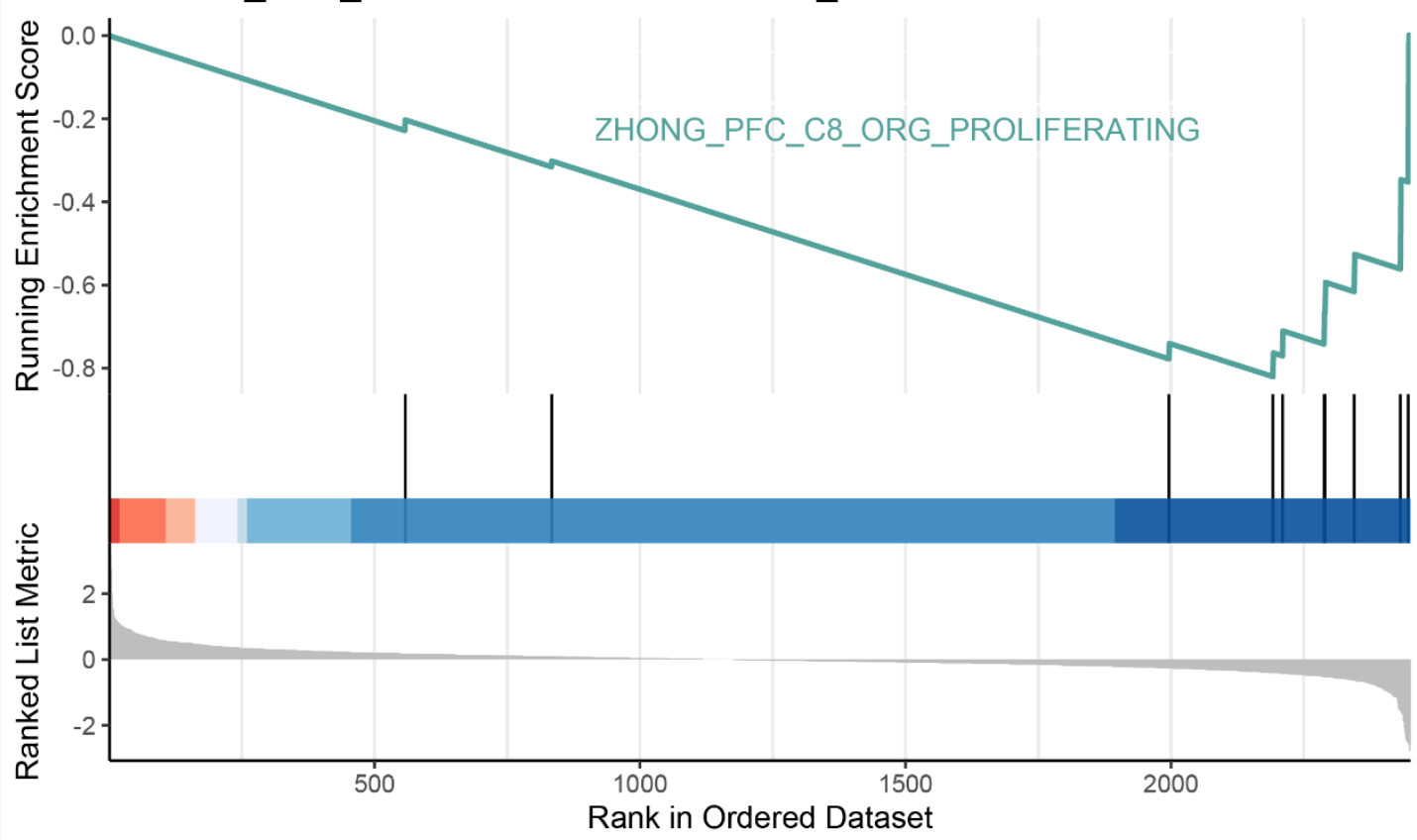

f GSEA of proteins that were highly expressed on the right side at GW 13. The analysis showed that the matrisome, as the main canonical pathway, was significantly affected by proteins expressed in the right frontal lobe (NES $=-0.71)$, and that proliferating ORG were significantly enriched in right frontal lobe samples (NES = $0.82)$. 
Fig. 5 
Clusters ordered based on number of genes and profiles ordered by significance (default)
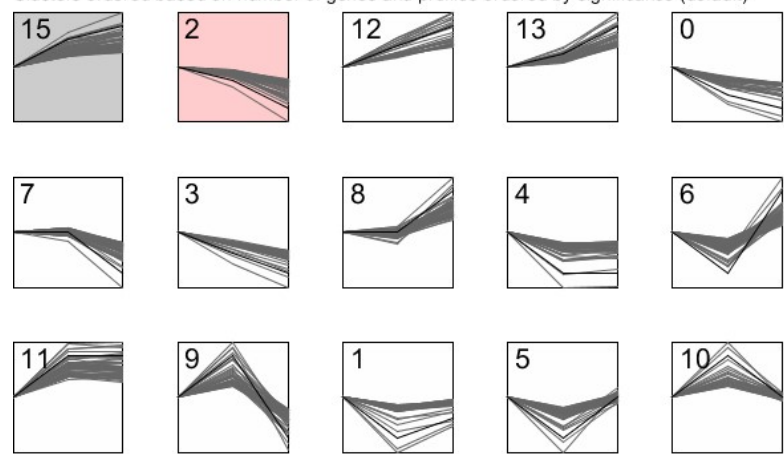
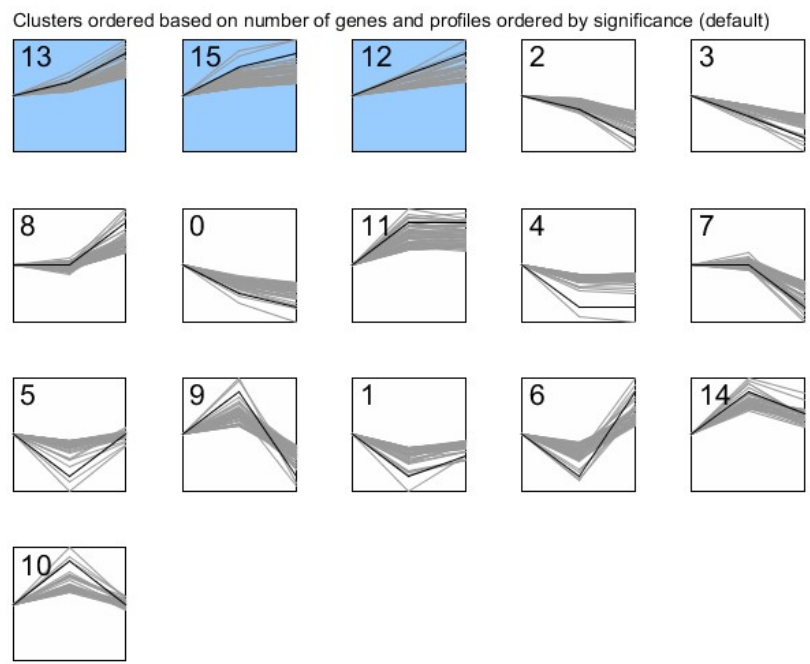

788 a Results of STEM analysis of core proteins in the left and right frontal lobes.

789 Significantly enriched clusters are indicated by different colours $(p<0.05)$. No

790 significant difference was found in the expression of clusters labeled white. 
B

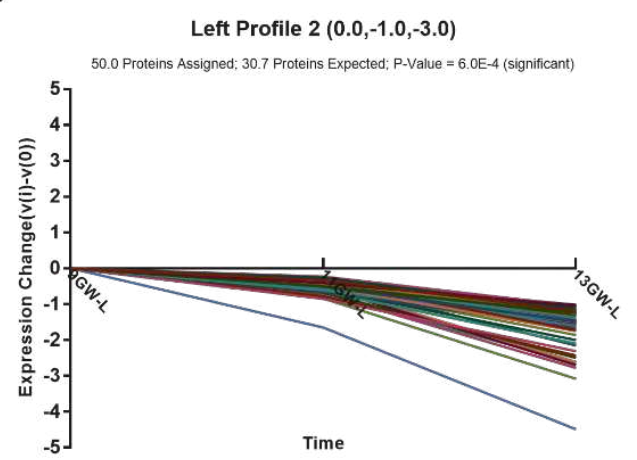

D

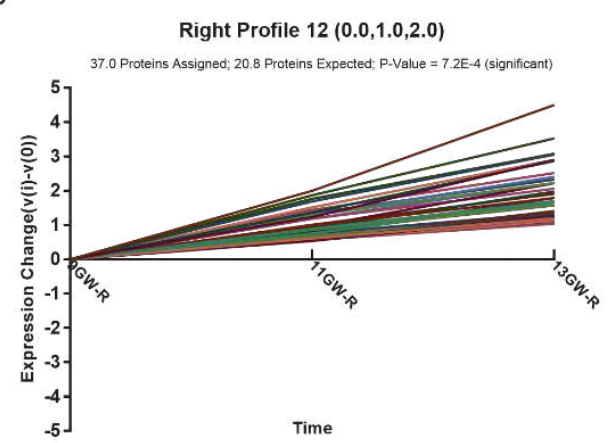

$\mathrm{F}$

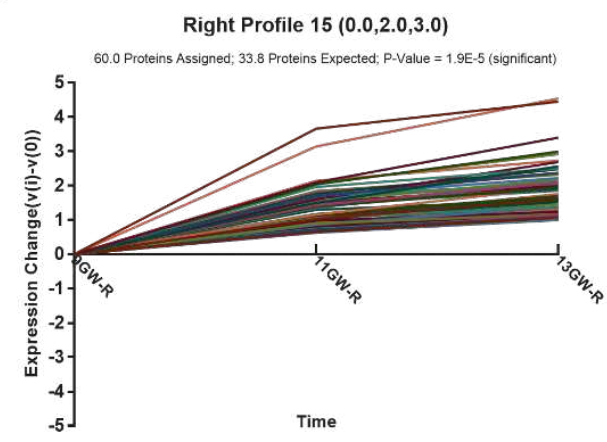

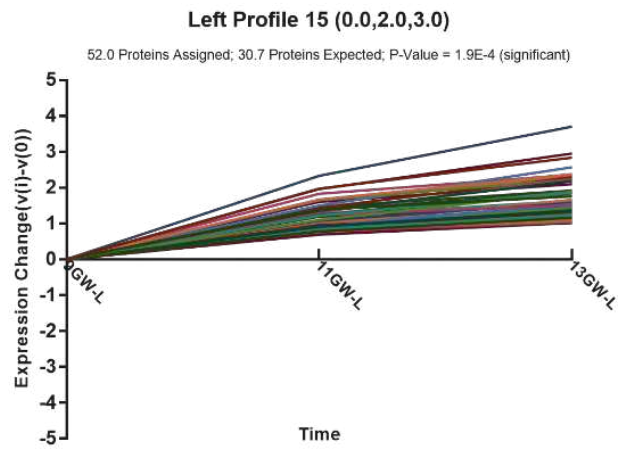

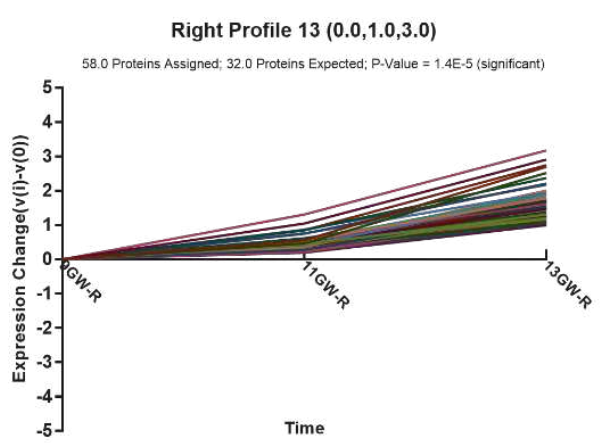

b The expression of cluster 2 proteins on the left side (50 assigned) was gradually downregulated, and the rate of downregulation decreased with increasing age. c The expression of cluster 15 proteins on the left side (52 assigned) was gradually upregulated, and the rate of upregulation decreased with increasing age. d The expression of cluster 12 proteins on the right side ( 37 assigned) was gradually upregulated with increasing age. 
799 e The expression of cluster 13 proteins on the right side (58 assigned) was gradually 800 upregulated, and the rate of upregulation increased with increasing age.

801 f The expression of cluster 15 proteins on the right side (60 assigned) was gradually 802 upregulated, and the rate of upregulation decreased with increasing age.

803

804 Fig. 6 


\section{Supplementary Files}

This is a list of supplementary files associated with this preprint. Click to download.

- SupplementaryTableLegends.docx

- SupplementaryFigure.docx

- SupplementaryTable.xlsx 